\title{
Subgroups of simple algebraic groups containing regular tori, and irreducible representations with multiplicity 1 non-zero weights
}

\author{
Donna Testerman and A.E. Zalesski
}

July 17, 2018

\begin{abstract}
Our main goal is to determine, under certain restrictions, the maximal closed connected subgroups of simple algebraic groups containing a regular torus. We call a torus regular if its centralizer is abelian. We also obtain some results of independent interest. In particular, we determine the irreducible representations of simple algebraic groups whose non-zero weights occur with multiplicity 1.
\end{abstract}

\section{Introduction}

Let $H$ be a simple algebraic group defined over an algebraically closed field $F$ of characteristic $p \geq 0$. The subgroups of $H$ containing a maximal torus are called subgroups of maximal rank. They play a substantial role in the structure theory of algebraic groups. A well-known and important classical result (going back to Dynkin and Borel-De Siebenthal) states that every subgroup of maximal rank is either contained in a parabolic subgroup of $H$, or lies in the normalizer of a subsystem subgroups (See [MT11, §13].) A subsystem subgroup $G$ is a semisimple subgroup of $H$, normalized by a maximal torus of $H$; consequently the root system of $G$ is, in a natural way, a subset of the root system of $H$. Note that Seitz Sei83 obtained some analog of the Dynkin-Borel-De Siebenthal classification for finite Chevalley groups. In addition, there are many results in the literature concerning classifying subgroups of Chevalley groups over infinite fields which contain a maximal split torus; for a discussion and bibliography see Vav95, Vav90.

Our aim here is to generalize the Dynkin-Borel-De Siebenthal result by replacing a maximal torus by a regular torus, that is, a torus whose centralizer in $H$ is a maximal torus of $H$. If $G$ is a closed subgroup of $H$ which contains a regular torus of $H$, then the maximal tori of $G$ are regular in $H$. Hence, we would like to determine the closed connected subgroups $G$ of $H$ whose maximal tori are regular in $H$. In its most general form, the question as stated is intractable; one 
has simply to think about indecomposable representations of semisimple groups to see that even in the case where $H$ is of type $A_{m}$, a complete classification is not realizable at this time, at least when $\operatorname{char}(F)>0$. A tractable version of the above question, and the one which we consider here is the following:

Problem 1. Determine (up to conjugacy) the maximal closed connected subgroups $G$ of a simple algebraic group $H$ containing a regular torus of $H$.

A reader familiar with the strategy for the study of subgroups of simple algebraic groups may raise the natural question: to what extent does the classification of the maximal closed connected subgroups of simple algebraic groups help in solving Problem 1. For example, can one simply "inspect" a list of maximal closed connected subgroups and arrive at the answer to the problem? We claim that this is not possible. Indeed, consider for example the case where $H$ is a classical group with natural module $V$. Here the classification of maximal closed connected subgroups reduces to a question about the maximality of simple subgroups $G \subset H$ acting irreducibly and tensor-indecomposably on $V$. Since the overwhelming majority of irreducible tensor-indecomposable subgroups of $H$ are maximal among closed connected subgroups of $H$, the classification consists of listing explicitly the non-maximal such subgroups. (See [Sei87, Thm. 3].) Therefore, it is impossible to decide by simple examination of maximal subgroups $G$ of $H$ which of them contains a regular torus of $H$, at least for classical groups $H$. The situation for the exceptional algebraic groups $H$ is somewhat different and will be discussed later.

The solution to Problem 1 must include of course all subgroups of $H$ of maximal rank. As mentioned above, such subgroups are either non reductive and therefore equal to a maximal parabolic subgroup (by [BT71]), or are described by the Dynkin-Borel-De Siebenthal classification. Therefore, we will henceforth consider reductive subgroups $G$ with $\operatorname{rank}(G)<\operatorname{rank}(H)$.

We use different approaches to deal with Problem 1 depending on whether $H$ is of classical or of exceptional type. In the former case our strategy is to reduce Problem 1 to the recognition of linear representations of simple algebraic groups $G$ whose weights satisfy certain specified properties. Denote by $V$ the natural module for $H$. Suppose first that $H$ is of type $A_{m}$. Then the embedding $G \rightarrow H$ can be viewed as a representation, and the condition for a torus $T$ of $G$ to be a regular torus in $H$ can be expressed in geometric terms. Specifically, using the language of representation theory, $T$ is a regular torus in $H$ if and only if all $T$-weight spaces of $V$ are one-dimensional. Therefore, one needs to determine the representations of simple algebraic groups all of whose weight spaces are onedimensional. In this form, the problem we are discussing was considered in a fundamental manuscript of Seitz [Sei87], which was the first major step toward extending Dynkin's classification of the maximal closed connected subgroups of the simple algebraic groups defined over $\mathbb{C}$ to the groups defined over fields of positive characteristic. For his purposes, Seitz only needed infinitesimally irreducible representations satisfying the condition on weight spaces; his result was later extended to general irreducible representations in [ZS87. (We quote 
the result in Proposition 3.1 below.)

Now suppose that $H$ is a classical group not of type $A_{m}$; again we view the embedding $G \rightarrow H$ as a representation of $G$. As $G$ is assumed to be maximal among closed connected subgroups, and not containing a maximal torus of $H$, a direct application of [Sei87, Thm. 3] shows that either $G$ acts irreducibly on $V$ or the pair $(G, H)$ is $\left(B_{m-k} B_{k}, D_{m+1}\right)$, for some $0 \leq k \leq m$. In the latter case, it is straightforward to show that $G$ contains a regular torus of $H$. See Section 2 for details.

Turning now to the irreducible subgroups $G \subset H$ containing a regular torus of $H$, we see that it is easier to determine such subgroups when $H$ is of type $B_{m}$ or $C_{m}$ due to the fact that a regular torus of $H$ is regular in $\mathrm{SL}(V)$. Hence, we may refer to the previously mentioned classification of representations having 1-dimensional weight spaces. If $H=D_{m}$, we need the following more precise result, which we prove in Section 4. Recall that the multiplicity of a weight in a representation is the dimension of the corresponding weight space.

Proposition 1.1. Let $H$ be a classical type simple algebraic group over $F$. Let $V$ be the natural FH-module and let $T$ be a (not necessarily maximal) torus in $H$. Then the following statements are equivalent:

(1) $T$ is a regular torus in $H$;

(2) Either all $T$-weights on $V$ have multiplicity 1 , or $H$ is of type $D_{m}$, and exactly one $T$-weight has multiplicity 2 and all other $T$-weights have multiplicity 1.

The above proposition motivates our consideration of irreducible representations of simple algebraic groups $G$ having at most one $T$-weight space of dimension greater than 1 (where $T$ is now taken to be a maximal torus of $G$ ). This is another main theme of this paper which contributes to the study of the pattern of weight multiplicities of irreducible representations of simple algebraic groups. This topic has been studied from various points of view, see for example [Sei87, BOS08, SZ92, Pre87]. In [BBL97, Propositions 3.4 and 3.6] Benkart, Britten and Lemire determined infinite-dimensional modules for the complex simple Lie algebras having a weight space decomposition with all weight spaces of dimension 1. Their results hint at some possible connection between weight multiplicities in irreducible representations of semisimple groups defined over a field of positive characteristic and weight multiplicities in appropriate infinitedimensional modules for semisimple complex Lie algebras.

Before stating our main result in this direction, we introduce the following notation. For a dominant weight $\lambda \in X(T)$, the group of rational characters of $T$, we denote by $L_{G}(\lambda)$ the irreducible $F G$-module of highest weight $\lambda$.

Theorem 1.2. Let $G$ be a simple algebraic group over $F$ and let $T \subset G$ be a maximal torus.

(1) Let $\lambda \in X(T)$ be a non-zero dominant weight. If $\operatorname{char}(F)=p>0$, assume in addition that $\lambda$ is p-restricted. All non-zero weights of $L_{G}(\lambda)$ are of multiplicity 1 if and only if $\lambda$ is as in Table 1 or Table 2. 
(2) Let $\mu \in X(T)$ be a non-zero dominant weight such that all non-zero weights of $L_{G}(\mu)$ are of multiplicity 1 . Then either all weights of $L_{G}(\lambda)$ have multiplicity 1 , or $\mu=p^{k} \lambda$ for some integer $k \geq 0$ and $\lambda$ as in Table 1 or Table 2, where $k=0$ if $\operatorname{char}(F)=0$.

In order to interpret the above-mentioned tables, we require the following definition.

Definition 1.3. Let $G$ be a semisimple algebraic group with maximal torus $T$. We denote by $\Omega_{2}(G)$ the set of p-restricted weights $\lambda \in X(T)$ such that all non-zero weights of $L_{G}(\lambda)$ have multiplicity 1 , and by $\Omega_{1}(G)$ the set of weights $\lambda \in \Omega_{2}(G)$ such that all weights of $L_{G}(\lambda)$ have multiplicity 1.

It is perhaps worth pointing out that there are no infinite families of examples in Table 2, unlike the situation with modules all of whose weight multiplicities are 1. Moreover, the set of weights $\Omega_{2}(G) \backslash \Omega_{1}(G)$ is independent of the characteristic; hence one has the same list in characteristic 0 and in positive characterisic $p$. Note that Table 2 also contains the data on the multiplicity of the zero weight in $L_{G}(\lambda)$. We think that this result may be useful for other applications.

In applying the above result to obtain a solution to Problem 1 for a classical type group $H$, we observe that certain irreducible representations $\rho_{1}, \rho_{2}$ of a simple group $G$, with highest weights $\lambda_{1}$, respectively $\lambda_{2}$, give rise to the same subgroup of $H$; that is, we find that $\rho_{1}(G)=\rho_{2}(G)$. For example, this happens for $\lambda_{2}=p^{k} \lambda_{1}$, or if $\rho_{1}$ is obtained from $\rho_{2}$ by applying a graph automorphism of $G$. In particular, this occurs for certain pairs $\lambda_{1}, \lambda_{2}$ such that all non-zero weights of $L_{G}\left(\lambda_{1}\right)$ and $L_{G}\left(\lambda_{2}\right)$ occur with multiplicity at most one. Such coincidences are clear and so we will not list them explicitly.

We now consider the image of the simple group $G$ under an irreducible representation $\rho$ having highest weight as in Theorem 1.2. To decide whether $\rho(G)$ contains a regular torus of the corresponding classical group, we must determine whether $\rho(G)$ stabilizes a non-degenerate quadratic or alternating form on the associated module, and apply Proposition 1.1. This will be carried out in Section 5 for tensor-indecomposable representations. See Section 2 for a discussion of the general case.

Now we turn to the case where $H$ is of exceptional Lie type. In contrast with the classical group case, the classification of maximal positive-dimensional closed subgroups of $H$ is explicit [LS04, Table 1]; we analyse the maximal connected subgroups which are not of maximal rank, and decide for which of these a maximal torus is regular in $H$. This is done in Proposition 6.1, Here our method uses a different aspect of representation theory than that used in the classical group case. It is based upon the following fact.

Proposition 1.4. Let $H$ be a connected algebraic group over $F$, with Lie algebra Lie $(H)$. Let $T \subset H$ be a (not necessarily maximal) torus. The torus $T$ is regular in $H$ if and only if $\operatorname{dim} C_{\operatorname{Lie}(H)}(T)=\operatorname{rank}(H)$.

This follows from the fact that $\operatorname{Lie}\left(C_{H}(S)\right)=C_{\operatorname{Lie}(H)}(S)$, for any torus $S \subset$ $H$. (See [Hum75, Prop. A. 18.4]). Now $T$ lies in a maximal torus $T_{H}$ of $H$ and 
so $\operatorname{Lie}\left(T_{H}\right)=C_{\operatorname{Lie}(H)}\left(T_{H}\right) \subset C_{\operatorname{Lie}(H)}(T)$. Hence, $T$ is regular in $H$ if and only if $C_{\operatorname{Lie}(H)}(T)=\operatorname{Lie}\left(T_{H}\right)$. If $G$ is explicitly given as a subgroup of $H$, one can determine the composition factors of the restriction of $\operatorname{Lie}(H)$ as $F G$-module; indeed this information is available in [LS04]. Next, for every composition factor, we determine the multiplicity of the zero weight. Then $C_{\operatorname{Lie}(H)}(T)=\operatorname{Lie}\left(T_{H}\right)$ if and only if the sum of these multiplicities equals the rank of $H$. Our solution to Problem 1 is explicit and the result is given in Table 10.

In contrast, for classical groups the main ingredient is the following statement:

Theorem 1.5. Let $G$ be a simple algebraic group over $F$ and $\rho: G \rightarrow \operatorname{GL}(V)$ an irreducible rational representation with p-restricted highest weight $\lambda$. Let $H \subset \mathrm{GL}(V)$ be the smallest simple classical group containing $\rho(G)$ and assume $\rho(G)$ contains a regular torus of $H$. Then the pairs $(G, \lambda)$ are those appearing in Tables 4, 5, 6, 7, 8, 9.

Observe that Theorem 1.5 includes the case of $\operatorname{char}(F)=0$. Moreover, we do not require $\rho(G)$ to be maximal in $H$. The specific case of maximal subgroups of classical type groups is treated in Theorems 5.6 and 5.7 .

The main result for subgroups of exceptional type groups $H$ does, however, require the hypothesis of maximality.

Theorem 1.6. Let $H$ be an exceptional simple algebraic group over $F$ and $M \subset$ $H$ a maximal closed connected subgroup containing a regular torus of $H$. Then either $M$ contains a maximal torus of $H$ or the pair $(M, H)$ is as in in Table 10.

Next we outline some possible applications of the above results.

We expect to use our results for recognition of linear groups, and more generally, for recognition of subgroups of algebraic groups that contain an element of a specific nature. This is the principal motivation for our consideration of Problem 2 below. A semisimple element of $H$ is regular if it centralizes no nontrivial unipotent element. (See Definition 1.8 and the discussion following the definition.) We now apply the following standard result.

Lemma 1.7. Hum75, Prop. 16.4] Let $K$ be a connected algebraic group. A torus $S$ of $K$ is regular if and only if $S$ contains a regular element.

As every semisimple element of $G$ belongs to a torus in $G$, Problem 1 is therefore equivalent to Problem 2.

Problem 2. Let $H$ be a simple algebraic group over $F$. Determine (up to conjugacy) all maximal closed connected subgroups $G$ of $H$ such that $G$ contains a regular semisimple element of $H$.

In order to place problem 2 in a more general context, let us recall a few well-known "recognition" results. Historically, Mitchell's classification of finite irreducible primitive linear groups over the complex numbers generated by reflections (1914) was among the most striking. Much more recently, Wagner 
Wag80, Wag81 and Zalesskiï-Serežkin [ZS80] classified irreducible linear groups over finite fields generated by reflections. In [Her74, Hering determined the subgroups of $G L(n, q)$ generated by irreducible elements of prime order.

Another potential application of our result is to the study of maximal subgroups of finite simple groups of Lie type, containing a regular element (of the ambient algebraic group). This problem was explicity stated by Walter in Wal90]. A related problem is studied in [SZ98, SZ00. There the authors analyze the irreducible $p$-modular representations of finite Chevalley groups $\rho: G \rightarrow H=G L(V)$ whose image contains a regular semisimple element of $H$, in the case where $p=\operatorname{char}(F)$ is the defining characteristic of $G$.

There is a series of results on recognition of finite linear groups containing a unipotent element of particular shape. Pollatsek [Pol76] in characteristic 2, and Wagner Wag78] and Zalesskii-Serežkin [ZS76] for odd characteristic, obtained a classification of irreducible linear groups over finite fields generated by transvections. Next Kantor [Kan79] classified the subgroups of finite classical groups $H$ generated by long root elements of $H$. In Coo81a, Coo81b, Cooperstein considered the case of finite groups of exceptional Lie type. In fact, Wagner also treated indecomposable subgroups, whereas Kantor and Cooperstein assumed that the subgroup under consideration has no normal unipotent subgroup.

Thompson Tho71 initiated the study of finite irreducible linear groups generated by elements $x$ with $(x-\mathrm{Id})^{2}=0$ (such elements are often called "quadratic"). Irreducible representations of simple algebraic groups whose image contains a quadratic unipotent element were determined by Premet and Suprunenko in PS83. Closed simple subgroups of simple algebraic groups containing long root elements were identified in Liebeck and Seitz LS94, where they also consider quasisimple subgroups of the finite groups arising as fixed points of some endomorphism of the algebraic group.

A result of a similar nature would be that of classifying linear groups $G$ over a finite field generated by regular unipotent elements, in place of root elements as in the above publications. (See Definition 1.8 below.) The case where $G$ is a connected algebraic group is a generic version of this problem. This has been considered by Suprunenko in Sup95. The case where $H$ is an arbitary simple algebraic group (in place of a special linear group) was considered by Saxl and Seitz [SS97] and the present authors [TZ13]. In [Sup95, $G$ is assumed to be an irreducible subgroup of the special linear group, in [SS97] $G$ is assumed to be maximal among positive-dimensional closed subgroups of an arbitrary simple algebraic group $H$, whereas in [TZ13], we assume that $G$ is connected reductive. Note that restrictions of this kind are unavoidable, as otherwise an explicit classification is impossible. The results in [SS97] and [TZ13] can be viewed as a kind of mirror image of those by Kantor and Cooperstein, in Kan79, Coo81a,

A general principle unifying the above results is to recognize a subgroup of a simple algebraic group by a conveniently verifiable property of a single element of the subgroup. That is, given a single element $g$ of $H$ described in convenient terms, determine the closed subgroups $G$ of $H$ containing $g$.

Following the above logic, we are concerned here with classifying the con- 
nected reductive algebraic subgroups of $H$ generated by regular elements, with further potential applications to finite algebraic groups. We recall the following

Definition 1.8. An element $x$ of a connected reductive algebraic group $H$ is said to be regular if $\operatorname{dim} C_{H}(x)=\operatorname{rank}(H)$.

Note that for a semisimple element $x \in H$, this is equivalent to saying that $C_{H}(x)^{\circ}$ is a torus, or equivalently that $C_{H}(x)$ contains no non-identity unipotent element. (See [SS70, Cor. 4.4] and [MT11, 14.7].) This is also equivalent to saying that $C_{H}(x)$ has an abelian normal subgroup of finite index.

In contrast with regular unipotent elements, the set of regular semisimple elements in $H$ contains infinitely many $H$-conjugacy classes. This suggests that the classification problem for subgroups containing such elements lies on a much higher level of difficulty. Nonetheless, our results lead to some progress in this direction.

It is of course natural to consider Problem 2 for arbitrary regular elements (i.e., not necessarily semisimple), especially because as mentioned above, the case where $g$ is a regular unipotent element was already studied and solved in [SS97, [TZ13, and Sup95. The following result is proven in Section 7 .

Theorem 1.9. Let $H$ be a connected reductive group over $F$. Let $G \subset H$ be a closed connected reductive subgroup. If $G$ contains a regular element of $H$ then $G$ contains a regular semisimple element of $H$.

The resolution of Problem 2 for arbitrary regular elements is reduced, by Theorem 1.9, to inspecting the subgroups $G$ of $H$ that contain a regular semisimple element of $H$. As mentioned above, the case of regular unipotent elements has been studied and resolved in [SS97, TZ13, while in the current manuscript, we treat the case of regular semisimple elements. To single out these cases is rather natural, but we do not think it particularly informative to analyse in detail other cases where $g$ is regular but neither semisimple nor unipotent. We indicate after the proof of Theorem 1.9 how one can handle the general case in terms of knowledge of the cases where $g$ is either semisimple or unipotent.

Notation. We fix here the notation and terminology to be used throughout the paper. We write $\mathbb{N}$ for the set of non-negative integers. Let $F$ be an algebraically closed field, of characteristic 0 or of prime characteristic $\operatorname{char}(F)=p>0$. For a natural number $a \geq 1$, we write $p \geq a$ (respectively $p>a$ ) to mean that either $\operatorname{char}(F)=0$ or $\operatorname{char}(F)=p \geq a($ resp. $\operatorname{char}(F)=p>a)$. For a linear algebraic group $X$ defined over $F$, we write $X^{\circ}$ for the connected component of the identity. All groups considered will be linear algebraic groups over $F$, and we will not repeat this each time.

Let $G$ be a reductive algebraic group over $F$. All $F G$-modules are assumed to be rational, and we will not make further reference to this fact. We fix a maximal torus and Borel subgroup $T \subset B$ of $G$, the root system $\Phi(G)$ with respect to $T$, a set of simple roots $\left\{\alpha_{1}, \ldots, \alpha_{n}\right\}$ corresponding to $B$, and the corresponding fundamental dominant weights $\left\{\omega_{1}, \ldots, \omega_{n}\right\}$. Write $X(T)$ for the 


\begin{tabular}{|l|c|}
\hline \multicolumn{1}{|c|}{$G$} & $\Omega_{1}(G) \backslash\{0\}$ \\
\hline \hline$A_{1}$ & $a \omega_{1}, 1 \leq a<p$ \\
\hline$A_{n}, n>1$ & $a \omega_{1}, b \omega_{n}, 1 \leq a, b<p$ \\
& $\omega_{i}, 1<i<n$ \\
& $c \omega_{i}+(p-1-c) \omega_{i+1}, 1 \leq i<n, 0 \leq c<p$ \\
\hline$B_{n}, n>2, p>2$ & $\omega_{1}, \omega_{n}$ \\
\hline$C_{n}, n>1, p=2$ & $\omega_{1}, \omega_{n}$ \\
\hline$C_{2}, p>2$ & $\omega_{1}, \omega_{2}, \omega_{1}+\frac{p-3}{2} \omega_{2}, \frac{p-1}{2} \omega_{2}$ \\
\hline$C_{3}$ & $\omega_{3}$ \\
\hline$C_{n}, n>2, p>2$ & $\omega_{1}, \omega_{n-1}+\frac{p-3}{2} \omega_{n}, \frac{p-1}{2} \omega_{n}$ \\
\hline$D_{n}, n>3$ & $\omega_{1}, \omega_{n-1}, \omega_{n}$ \\
\hline$E_{6}$ & $\omega_{1}, \omega_{6}$ \\
\hline$E_{7}$ & $\omega_{7}$ \\
\hline$F_{4}, p=3$ & $\omega_{4}$ \\
\hline$G_{2}, p \neq 3$ & $\omega_{1}$ \\
\hline$G_{2}, p=3$ & $\omega_{1}, \omega_{2}$ \\
\hline
\end{tabular}

Table 1: Irreducible $p$-restricted $G$-modules with all weights of multiplicity 1

group of rational characters on $T$. Given a dominant weight $\lambda \in X(T)$, we write $L_{G}(\lambda)$ for the irreducible $F G$-module with highest weight $\lambda$. A dominant weight $\lambda \in X(T)$ is said to be $p$-restricted if either $\operatorname{char}(F)=0$ or $\operatorname{char}(F)=p$ and $\lambda=\sum a_{i} \omega_{i}$ with $a_{i}<p$ for all $i$. Recall that a weight $\mu \in X(T)$ is said to be subdominant to $\lambda$ if $\lambda$ and $\mu$ are dominant weights and $\mu=\lambda-\sum a_{i} \alpha_{i}$ for some $a_{i} \in \mathbb{N}$. For an $F G$-module $M$ and a weight $\mu \in X(T)$, we let $M_{\mu}$ denote the $T$-weight space corresponding to the weight $\mu$. Set $W_{G}:=N_{G}(T) / T$, the Weyl group of $G$ and write $s_{i}$ for the reflection in $W_{G}$ corresponding to the simple root $\alpha_{i}$. We label Dynkin diagrams as in Bourbaki [Bou68].

We will assume that $p>2$ when $G$ is simple of type $B_{n}$, since for our purposes, when $p=2$ the group $B_{n}$ can be treated as a group of type $C_{n}$. When $G$ is a classical type simple algebraic group, by the "natural" module for $G$ we mean $L_{G}\left(\omega_{1}\right)$. If $G$ is simple of type $B_{n}, C_{n}$, or $D_{n}$, it is well-known that $G$ preserves a non-degenerate bilinear or quadratic form on the natural module.

\section{Initial reductions}

Let $H$ be a simple algebraic group over $F$ and let $G$ be a maximal closed connected subgroup of $H$ which contains a regular torus of $H$. If $G$ is not reductive, then [BT71] implies that $G$ is a maximal parabolic subgroup of $H$ and hence contains a maximal torus of $H$. Henceforth we will restrict our attention to reductive subgroups $G$.

Consider now the case where $H$ is a classical type simple algebraic group with natural module $V$. We use the general reduction theorem, Sei87, Thm. 


\begin{tabular}{|c|c|c|c|}
\hline$G$ & conditions & $\Omega_{2}(G) \backslash \Omega_{1}(G)$ & weight 0 multiplicity \\
\hline$A_{n}$, & $n>1,(n, p) \neq(2,3)$ & $\omega_{1}+\omega_{n}$ & $\begin{cases}n & \text { if } p \nmid(n+1) \\
n-1 & \text { if } p \mid(n+1)\end{cases}$ \\
\hline$A_{3}$ & $p>3$ & $2 \omega_{2}$ & 2 \\
\hline \multirow[t]{2}{*}{$\overline{B_{n}}$} & \multirow[t]{2}{*}{$n>2, p>2$} & $\omega_{2}$ & $n$ \\
\hline & & $2 \omega_{1}$ & $\begin{cases}n & \text { if } p \mid(2 n+1) \\
n+1 & \text { if } p \nmid(2 n+1)\end{cases}$ \\
\hline \multirow[t]{2}{*}{$\overline{C_{n}}$} & $n>1$ & $2 \omega_{1}$ & $n$ \\
\hline & $n>2,(n, p) \neq(3,3)$ & $\omega_{2}$ & $\begin{cases}n-2 & \text { if } p \mid n \\
n-1 & \text { if } p \not x n\end{cases}$ \\
\hline$C_{2}$ & $p \neq 5$ & $2 \omega_{2}$ & 2 \\
\hline$C_{4}$ & $p \neq 2,3$ & $\omega_{4}$ & 2 \\
\hline$D_{n}$ & $n>3, p>2$ & $2 \omega_{1}$ & $\begin{cases}n-2 & \text { if } p \mid n \\
n-1 & \text { if } p \nmid n\end{cases}$ \\
\hline$D_{n}$ & $n>3, p=2$ & $\begin{array}{l}\omega_{2} \\
\omega_{2} \\
\end{array}$ & $\begin{array}{c}n \\
n-\operatorname{gcd}(2, n)\end{array}$ \\
\hline \multirow{2}{*}{$E_{6}$} & & \multirow{2}{*}{$\omega_{2}$} & $\{5 \quad$ if $p=3$ \\
\hline & & & $6 \quad$ if $p \neq 3$ \\
\hline \multirow{2}{*}{$E_{7}$} & & \multirow{2}{*}{$\omega_{1}$} & $\int 7$ if $p \neq 2$ \\
\hline & & & $6 \quad$ if $p=2$ \\
\hline$E_{8}$ & & $\omega_{8}$ & 8 \\
\hline \multirow{2}{*}{$F_{4}$} & & \multirow{2}{*}{$\omega_{1}$} & 2 if $p=2$ \\
\hline & $p \neq 3$ & & $\begin{array}{l}4 \quad \text { if } p \neq 2 \\
2\end{array}$ \\
\hline$G_{2}$ & $p \neq 3$ & $\omega_{2}$ & 2 \\
\hline
\end{tabular}

Table 2: Irreducible $p$-restricted $G$-modules with non-zero weights of multiplicity 1 and whose zero weight has multiplicity greater than 1 . 
3], on maximal closed connected subgroups of $H$; this allows us to restrict our considerations to irreducibly acting subgroups of $H$. For a detailed discussion of this see [MT11, §18].

Let $G$ be maximal among closed connected subgroups. Then one of the following holds:

1. $G$ contains a maximal torus of $H$.

2. $H$ is of type $D_{m}, V=U \oplus U^{\perp}, U$ is an odd-dimensional non-degenerate subspace with respect to the bilinear form on $V, 2 \leq \operatorname{dim} U \leq \operatorname{dim} V-2$, and $G=\operatorname{Stab}_{H}(U)^{\circ}$.

3. $V=V_{1} \otimes V_{2}$, each of $V_{1}$ and $V_{2}$ is equipped with either the zero form (in case $V$ has no non-degenerate $H$-invariant form), or a non-degenerate bilinear or quadratic form, and the form on $V$ is obtained as the product form. Moreover, $G$ is the connected component of $\left(\operatorname{Isom}\left(V_{1}\right) \otimes \operatorname{Isom}\left(V_{2}\right)\right) \cap H$. Note that if $V$ is equipped with a quadratic form and $\operatorname{char}(F)=2$, then $G=\operatorname{Sp}\left(V_{1}\right) \otimes \operatorname{Sp}\left(V_{2}\right)$.

4. $G$ is simple acting irreducibly and tensor indecomposably on $V$.

In the first three cases, it is straightforward to show that $G$ contains a regular torus of $H$. Hence we are reduced to considering simple subgroups which act irreducibly and tensor indecomposably on $V$.

\section{Irreducible representations of simple algebraic groups whose non-zero weights are of multiplicity 1}

In this section, we obtain the classification of irreducible representations all of whose non-zero weights have multiplicity 1 , thereby establishing Theorem 1.2 . Throughout this section we take $G$ to be a simply connected simple algebraic group over $F$. The rest of the notation will be as fixed in Section 1, As discussed in Section 1, we will require the following classification.

Proposition 3.1. Let $\lambda \in X(T)$ be a non-zero dominant weight.

(1) Assume in addition that $\lambda$ is p-restricted. Then all weights of $L_{G}(\lambda)$ are of multiplicity 1 if and only if $\lambda$ is as in the second column of Table 1 . In other words, the set $\Omega_{1}(G) \backslash\{0\}$ is as given in Table 1 .

(2) Suppose that $p>0$ and $\lambda$ is not p-restricted, so $\lambda=\sum_{i=0}^{k} p^{i} \lambda_{i}$, where $\lambda_{i}$ are $p$-restricted and $\lambda_{i} \neq 0$ for some $i>0$. Then all weights of $L_{G}(\lambda)$ are of multiplicity 1 if and only if the following hold:

(a) for all $0 \leq l \leq k, \lambda_{l}=0$ or $\lambda_{l} \in \Omega_{1}(G)$, and 
(b) for all $0 \leq l<k$,

$$
\begin{aligned}
& \left(\lambda_{l}, \lambda_{l+1}\right) \neq\left(\omega_{n}, \omega_{1}\right) \text { if } p=2, G=C_{n} ; \\
& \left(\lambda_{l}, \lambda_{l+1}\right) \neq\left(\omega_{1}, \omega_{1}\right) \text { if } p=2, G=G_{2} ; \\
& \left(\lambda_{l}, \lambda_{l+1}\right) \neq\left(\omega_{2}, \omega_{1}\right) \text { if } p=3, G=G_{2} .
\end{aligned}
$$

Proof. The result follows from [Sei87, 6.1] and [ZS87, Prop.2].

We now collect some results on dimensions of certain weight spaces in infinitesimally irreducible $F G$-modules. The proofs can be found in [Sei87], Tes88] and $[\mathrm{BGT}$.

Lemma 3.2. Let $\lambda \in X(T)$ be a p-restricted dominant weight.

(1) If $G$ is of type $A_{n}$ and $\lambda=a \omega_{j}+b \omega_{k}$, with $a b \neq 0$, then the multiplicity of the weight $\lambda-\alpha_{j}-\alpha_{j+1}-\cdots-\alpha_{k}$ in $L_{G}(\lambda)$ is $k-j+1$ unless $p \mid(a+b+k-j)$, in which case the multiplicity is $k-j$.

(2) If $G$ is of type $A_{n}$ and $\lambda=c \omega_{i}$ for some $1<i<n$ and $c>1$, then the multiplicity of the weight $\lambda-\alpha_{i-1}-2 \alpha_{i}-\alpha_{i+1}$ in $L_{G}(\lambda)$ is 2 unless $c=p-1$ in which case the multiplicity is 1 .

(3) If $G$ is of type $B_{2}$ and $\lambda=a \omega_{1}+b \omega_{2}$, with $a b \neq 0$, the multiplicity of the weight $\lambda-\alpha_{1}-\alpha_{2}$ in $L_{G}(\lambda)$ is 2 unless $2 a+b+2 \equiv 0(\bmod p)$ in which case the multiplicity is 1 .

(4) If $G$ is of type $B_{n}$ with $\lambda=\omega_{1}+\omega_{n}$, then the weight $\lambda-\alpha_{1}-\cdots-\alpha_{n}$ has multiplicity $n$ in $L_{G}(\lambda)$, unless $p \mid(2 n+1)$ in which case it has multiplicity $n-1$.

(5) If $G$ is of type $G_{2}$ and $\lambda=a \omega_{1}+b \omega_{2}$, with $a b \neq 0$, the multiplicity of the weight $\lambda-\alpha_{1}-\alpha_{2}$ in $L_{G}(\lambda)$ is 2 unless $3 a+b+3 \equiv 0(\bmod p)$ in which case the multiplicity is 1 .

(6) If $G$ is of type $A_{n}$ and $\lambda=a \omega_{i}+b \omega_{i+1}+c \omega_{i+2}$, with $a b c \neq 0$ and $a+b=$ $p-1=b+c$, the weight $\lambda-\alpha_{1}-\alpha_{2}-\alpha_{3}$ has multiplicity at least 2 in the module $L_{G}(\lambda)$.

(7) If $G$ is of type $D_{4}$ and $\lambda=a \omega_{1}$, with $a>1$, then the weight $\lambda-2 \alpha_{1}-2 \alpha_{2}-$ $\alpha_{3}-\alpha_{4}$ has multiplicity at least 2 in $L_{G}(\lambda)$.

(8) If $G$ is of type $G_{2}, \lambda=b \omega_{1}$ with $b>1$, and $p>3$, then the weight $\lambda-2 \alpha_{1}-\alpha_{2}$ has multiplicity 2 in $L_{G}(\lambda)$.

(9) If $G$ is of type $G_{2}, \lambda=a \omega_{2}$, with $p>3$ and $a=\frac{p-1}{2}$, then the weight $\lambda-2 \alpha_{1}-2 \alpha_{2}$ has multiplicity 2 in $L_{G}(\lambda)$.

Proof. Part (a) is [Sei87, 8.6], (c) and (e) are [Tes88, 1.35]. For (b), see the proof of [Sei87, 6.7] and apply the main result of [Pre87. Part (d) is [BGT, 2.2.7]. The proof of (f) is contained in the proof of [Sei87, 6.10]. Part (g) is proved in Sei87, 6.13]. Finally, the proofs of (h) and (i) follow from the proof in Sei87, $6.18]$. 
We now turn our attention to the determination of the set $\Omega_{2}(G) \backslash \Omega_{1}(G)$. Let $\lambda$ be a $p$-restricted dominant weight for the group $G$. It will be useful to work inductively, restricting the representation $L_{G}(\lambda)$ to certain subgroups and applying the following analogue of [Sei87, 6.4].

Lemma 3.3. Let $X$ be a subsystem subgroup of $G$ normalized by $T$. Let $\lambda \in$ $\Omega_{2}(G)$. Let $L_{X}(\mu)$ be an $F X$-composition factor of $L_{G}(\lambda)$, for some dominant weight $\mu$ in the character group of $X \cap T$. Then $\mu \in \Omega_{2}(X)$.

Proof. The argument is completely analogous to the proof of Sei87, Lemma 6.4]. Set $W:=L_{G}(\lambda)$. Write $T X=X Z$, where $Z=C_{T}(X)^{\circ}$. Let $0 \subset M_{1} \subset$ $\cdots \subset M_{t}=W$ be an $F(X T)$-composition series of $W$. Then there exists $i$ such that $L_{X}(\mu) \cong M_{i} / M_{i-1}$. Now $M_{i}=M_{i-1} \oplus M^{\prime}$ as $F T$-modules, $Z$ acts by scalars on $M^{\prime}$ and the set of $(T \cap X)$-weights in $M^{\prime}$ (and their multiplicities) are precisely the same as in $L_{X}(\mu)$. Also, if $\nu$ is a non-zero weight of $L_{X}(\mu)$, then $\nu$ corresponds to a non-zero $T$-weight of $M^{\prime}$. Therefore if $\nu$ is a $(T \cap X)$-weight occurring in $L_{X}(\mu)$ with multiplicity greater than 1 , there exists a $T$-weight $\nu^{\prime}$ such that $\operatorname{dim}\left(M^{\prime}\right)_{\nu^{\prime}} \geq 2$. So $\nu^{\prime}=0$ and hence $\nu=0$. The result follows.

Proposition 3.4. The set of weights $\Omega_{2}(G) \backslash \Omega_{1}(G)$ is as given in Table 2. Moreover, the multiplicity of the zero weight in $L_{G}(\lambda)$ is as indicated in the fourth column.

Proof. We first note that for $G$ and $\lambda$ as in Table 2, the multiplicity of the zero weight in $L_{G}(\lambda)$ can be deduced from [Lüb01, Table 2]. We now show that the list in Table 2 contains all weights in $\Omega_{2}(G) \backslash \Omega_{1}(G)$. Let $\lambda \in \Omega_{2}(G) \backslash \Omega_{1}(G)$; in particular, 0 must be subdominant to $\lambda$, and so $\lambda$ lies in the root lattice. We will proceed as in [Sei87, §6]. We apply Lemma 3.3 to various subsystem subgroups of $G$; all of such are taken to be normalized by the fixed maximal torus $T$.

Case $\mathbf{A}_{\mathbf{3}}$. Consider first the case where $\lambda=b \omega_{2}$. By the above remarks, $b>1$, and so we have $p>2$. If $b=2$, the only weights subdominant to $\lambda$ are $\lambda-\alpha_{2}$, which has multiplicity 1 in $L_{G}(\lambda)$ and $\mu=\lambda-\alpha_{1}-2 \alpha_{2}-\alpha_{3}$ which is the zero weight. Hence $\lambda \in \Omega_{2}(G)$ and by Lemma $3.2(2), \lambda \in \Omega_{1}(G)$ if and only if $p=3$. If $b>2$, the weight $\mu$ is a non-zero weight and Lemma 3.2(2) implies that $b=p-1$, in which case $\lambda \in \Omega_{1}(G)$.

Now consider the general case $\lambda=a \omega_{1}+b \omega_{2}+c \omega_{3}$. Assume for the moment that $a b c \neq 0$. Applying Lemma $3.2(1)$, we see that $a+b=p-1=c+d$. But then Lemma 3.2(6) rules out this possibility. Hence we must have $a b c=0$. If $a b \neq 0$ as above we have $a+b=p-1$ and $\lambda \in \Omega_{1}(G)$. The case $b c \neq 0$ is analogous. If $b=0$ and $a c \neq 0$, Lemma 3.2(1) implies that the weight $\lambda-\alpha_{1}-\alpha_{2}-\alpha_{3}$ must be the zero weight and hence $a=1=c$. This weight appears in Table 2 , Finally, if $b=c=0$ or $a=b=0$, then $\lambda \in \Omega_{1}(G)$. This completes the case $G=A_{3}$.

Case $\mathbf{A}_{\mathbf{n}}, \mathbf{n} \neq \mathbf{3}$. If $n=2$, Lemma 3.2(1) and Proposition 3.1 give the result. So we now assume $n>3$. Applying Lemma 3.3 to various $A_{3}$ Levi factors of $G$, as well as the result of Lemma $3.2(1)$ and (2), we are reduced to configurations of the form: 
a) $\lambda=\omega_{i}$ for some $i$,

b) $\lambda=(p-1) \omega_{i}$, for $1<i<n$,

c) $\lambda=a \omega_{i}, i=1, n$,

d) $\lambda=c \omega_{i}+d \omega_{i+1}, c d \neq 0,1 \leq i<n$ and $c+d=p-1$,

e) $\lambda=\omega_{1}+\omega_{n}$.

Each of these weights is included either in $\Omega_{1}(G)$ or in Table 2 .

Case $\mathbf{C}_{2}$. Let $\lambda=d \omega_{1}+c \omega_{2}$. The arguments of [Sei87, 6.11] together with Lemma 3.2 (3) show that either $\lambda \in \Omega_{1}(G)$ or one of the following holds:

(a) $d=0, c>1, c \neq \frac{p-1}{2}$, and the weight $\lambda-2 \alpha_{1}-2 \alpha_{2}$ is the zero weight.

(b) $c=0, d>1$, and the weight $\lambda-2 \alpha_{1}-\alpha_{2}$ is the zero weight.

(c) $c d \neq 0, d>1,2 c+d+2 \equiv 0(\bmod p)$ and $\lambda-\alpha_{1}-\alpha_{2}$ is the zero weight.

Case (i) is satisfied only if $c=2$; (ii) is satisfied only if $d=2$; (iii) is not possible.

Case $\mathbf{C}_{\mathbf{n}}, \mathbf{n}>\mathbf{2}$. First suppose $n=3$ and let $\lambda=a \omega_{1}+b \omega_{2}+c \omega_{3}$. We apply Lemma 3.3 to three different $C_{2}$ subsystem subgroups of $G$, namely $X_{1}$, the Levi factor corresponding to the set $\left\{\alpha_{2}, \alpha_{3}\right\}, X_{2}$, the conjugate of this group by the reflection $s_{1}$, and $X_{3}=X_{1}^{w}$, where $w=s_{1} s_{2}$. Restricting $\lambda$ to $X_{1}$ gives that

$$
(b, c) \in\left\{(0,0),(1,0),(2,0),(0,1),(0,2),\left(1, \frac{p-3}{2}\right),\left(0, \frac{p-1}{2}\right)\right\} .
$$

Note also that $\left.\lambda\right|_{T \cap X_{2}}$ has highest weight $(a+b) \mu_{1}+c \mu_{2}$, where $\mu_{1}, \mu_{2}$ are the fundamental dominant weights corresponding to the base $\left\{\alpha_{1}+\alpha_{2}, \alpha_{3}\right\}$.

Suppose first that $c \neq 0$, so $b \in\{0,1\}$. If $a+b<p$, we can again apply the $C_{2}$ result to the group $X_{2}$ to see that $a+b=0$ or 1 . On the other hand, if $a+b \geq p$, we must have $b=1$ and $a=p-1$. This latter case is not possible as Lemma 3.2(1) implies that the non-zero weight $\lambda-\alpha_{1}-\alpha_{2}$ has multiplicity 2. Hence when $c \neq 0$, we have $(a, b, c)=(0,0, c)$, or $(a, b, c)=(1,0, c)$, or $(a, b, c)=\left(0,1, \frac{p-3}{2}\right)$. As the third possibility corresponds to a weight in $\Omega_{1}(G)$, we consider the first two possibilities. If $(a, b, c)=(0,0, c)$, by Proposition 3.1, we may assume $c \neq \frac{p-1}{2}$, and $c \neq 1$. This leaves us with the weight $2 \omega_{3}$, and $p \neq 5$. But then Lüb01] shows that a non-zero weight has multiplicity greater than 1. If $(a, b, c)=(1,0, c)$, then $c \in\left\{1,2, \frac{p-1}{2}\right\}$. The restriction of $\lambda$ to the subgroup $X_{2}$ is $\mu_{1}+c \mu_{2}$. But here Lemma [3.2(3) shows that the weight $\mu=\lambda-\left(\alpha_{1}+\alpha_{2}\right)-\alpha_{3}$ has multiplicity 2 unless $c=\frac{p-3}{2}$. Since $\mu$ is a non-zero weight, either $c=1$ and $p=5$ or $c=2$ and $p=7$. Again, we refer to Lüb01 to see that there is a non-zero weight with multiplicity greater than one in each case.

Suppose now $c=0$. Then the restriction to $X_{1}$ implies that $(a, b, c)$ is one of $(a, 1,0),(a, 2,0),(a, 0,0)$. Suppose $(a, b, c)=(a, 1,0)$. If $a=0$, then 
the only subdominant weight in $L_{G}(\lambda)$ is the 0 weight and hence this gives an example. If $a \neq 0$, Lemma 3.2(1) implies that $a=p-2$. Then the restriction of $\lambda$ to $X_{3}$ is the weight $a \eta_{1}+\eta_{2}$, where $\eta_{1}, \eta_{2}$ are the fundamental dominant weights corresponding to the base $\left\{\alpha_{1}, 2 \alpha_{2}+\alpha_{3}\right\}$. But then Lemma 3.2(3) implies that the non-zero weight $\lambda-\alpha_{1}-2 \alpha_{2}-\alpha_{3}$ occurs with multiplicity 2 . If $(a, b, c)=(a, 2,0)$, then the subdominant weight $\lambda-2 \alpha_{2}-\alpha_{3}$ has multiplicity 2 and hence this is not an example. Finally, if $(a, b, c)=(a, 0,0)$, we consider the restriction of $\lambda$ to the subgroup $X_{3}$ and the $C_{2}$ result implies that $a=1$ or $a=2$. If $a=1$, then $\lambda \in \Omega_{1}(G)$, while if $a=2$, [Lüb01, Table 2] shows that $\lambda \in \Omega_{2}(G)$. This completes the consideration of the case $G=C_{3}$.

Consider now the general case where $n \geq 4$. If $\lambda=\sum a_{i} \omega_{i}$ with $a_{i}=0$ for $i \leq n-2$, then the $C_{3}$ result and Lemma 3.3 (applied to the standard $C_{3}$ Levi factor) implies that either $\lambda$ is one of the weights in Table 1 or in Table 2, or $\lambda=\omega_{n-1}$ or $\lambda=\omega_{n}$, with $p \neq 3$ in each case. If $\lambda=\omega_{n-1}$, then we refer to Lüb01, for the group $C_{4}$, to see that the subdominant weight $\omega_{n-3}$ occurs with multiplicity 2. This then shows that $\lambda=\omega_{n-1} \notin \Omega_{2}(G)$. Now if $\lambda=\omega_{n}$, again use [Lüb01] and find that $\lambda \in \Omega_{2}(G)$ when $n=4$, while if $n>4$, the weight $\omega_{n-4}$ occurs with multiplicity 2 and so $\lambda \notin \Omega_{2}(G)$.

We may now assume that there exists $i \leq n-2$ with $a_{i} \neq 0$. Choose $i \leq n-2$ maximal with $a_{i} \neq 0$ and consider the $C_{3}$ subsystem subgroup $X$ with root system base $\left\{\alpha_{i}+\cdots+\alpha_{n-2}, \alpha_{n-1}, \alpha_{n}\right\}$, so that $\left.\lambda\right|_{T \cap X}=a_{i} \eta_{1}+a_{n-1} \eta_{2}+a_{n} \eta_{3}$, where $\left\{\eta_{1}, \eta_{2}, \eta_{3}\right\}$ are the fundamental dominant weights corresponding to the given base. As $a_{i} \neq 0$, the $C_{3}$ case considerations imply that $a_{n-1}+a_{n}=0$ and $a_{i}=1$ or 2 . If $i=1, \lambda$ occurs in the statement of the result. If $i=2$, so $\lambda=a_{1} \omega_{1}+a_{2} \omega_{2}$, then we may assume $a_{1} \neq 0$, or $a_{2}=2$, as $\lambda=\omega_{2}$ occurs in the statement of the result. But then the restriction of $\lambda$ to the $C_{3}$ subsystem subgroup with root system base $\left\{\alpha_{1}, \alpha_{2}+\cdots+\alpha_{n-1}, \alpha_{n}\right\}$ has non-zero weights occurring with multiplicity greater than 1.

So finally, we may assume $i>2$, and so $n \geq 5$. If $n=5$ and so $i=3$, we apply the result for $C_{4}$ to see that $a_{2}=0$ and $a_{3}=1$. But the non-zero weight $\lambda-\alpha_{2}-2 \alpha_{3}-2 \alpha_{4}-\alpha_{5}$ has multiplicity at least 2 by the $C_{4}$ result. Hence the result holds for the case $n=5$. If $n \geq 6$, we consider the $C_{5}$ subsystem subgroup whose root system has base $\left\{\alpha_{i-2}, \alpha_{i-1}, \alpha_{i}+\cdots+\alpha_{n-2}, \alpha_{n-1}, \alpha_{n}\right\}$ and obtain a contradiction.

Case $\mathbf{D}_{\mathbf{n}}$. Suppose first that $n=4$. Let $\lambda=\sum a_{i} \omega_{i}$. If $a_{2}=0$, Lemma 3.2(1) implies that $\lambda=a_{i} \omega_{i}$ for $i=1,3$ or 4 ; assume by symmetry that $i=1$. Then Lemma 3.2(7) shows that either $a_{1}=1$ or $\lambda-2 \alpha_{1}-2 \alpha_{2}-\alpha_{3}-\alpha_{4}$ is the zero weight. In either case, $\lambda$ is as in the statement of the result. So we now assume $a_{2} \neq 0$. Using the result for $A_{3}$, applied to the three standard $A_{3}$ Levi factors, we see that at most one of $a_{1}, a_{3}, a_{4}$ is non-zero. However if $a_{1}+a_{3}+a_{4} \neq 0$, Lemma $3.2(1)$ and (2) provide a contradiction. Hence $\lambda=a_{2} \omega_{2}$, and since $\lambda=\omega_{2}$ is in Table 2, we may assume $a_{2}>1$. Now $\lambda-\alpha_{1}-2 \alpha_{2}-\alpha_{3}$ is a non-zero weight, so Lemma 3.2 (2) implies that $a_{2}=p-1$. But we now apply Lemma 3.3 to the $A_{3}$ subsystem subgroup with root system having as base $\left\{\alpha_{2}, \alpha_{1}, \alpha_{2}+\alpha_{3}+\alpha_{4}\right\}$ 
to obtain a contradiction.

Now consider the general case where $n>4$. We argue by induction on $n$. Apply the result for $D_{n-1}$ to the standard $D_{n-1}$ Levi factor of $G$ to see that either $\lambda$ is as in the statement of the result or $\lambda=a \omega_{1}, a \omega_{1}+\omega_{2}, a \omega_{1}+\omega_{n-1}, a \omega_{1}+\omega_{n}$, $a \omega_{1}+\omega_{3}$, or $a \omega_{1}+2 \omega_{2}$. Now consider the $D_{4}$ subsystem subgroup whose root system has base $\left\{\alpha_{1}, \alpha_{2}+\cdots+\alpha_{n-2}, \alpha_{n-1}, \alpha_{n}\right\}$. The result for $D_{4}$ then implies that either $\lambda$ appears in Table 1 or Table 2, or $\lambda=\omega_{3}$. But then the restriction of $\lambda$ to the standard $D_{n-1}$ Levi factor $X$ affords a composition factor which is the unique nontrivial composition factor of $\operatorname{Lie}(X)$. The weight corresponding to the zero weight in $\operatorname{Lie}(X)$ is the weight $\lambda-\alpha_{1}-2\left(\alpha_{2}+\cdots+\alpha_{n-2}\right)-\alpha_{n-1}-\alpha_{n}$, which is a non-zero weight in $L_{G}(\lambda)$ with multiplicity at least $n-3$. (See [Lüb01, Table 2].) This completes the consideration of type $D_{n}$.

Case $\mathbf{B}_{\mathbf{n}}$. As throughout the paper, we assume $p>2$ when $G$ has type $B_{n}$. Consider first the case $n=3$. We apply the result for $C_{2}$ to the standard $C_{2}$ Levi factor of $G$ and the $A_{3}$ result to the subsystem subgroup $X$ with root system base $\left\{\alpha_{2}+2 \alpha_{3}, \alpha_{1}, \alpha_{2}\right\}$. Note that if $\lambda=a \omega_{1}+b \omega_{2}+c \omega_{3}$, then the restriction of $\lambda$ to $X$ affords a composition factor with highest weight $(b+c) \eta_{1}+a \eta_{2}+b \eta_{3}$, where $\left\{\eta_{1}, \eta_{2}, \eta_{3}\right\}$ is the set of fundamental dominant weights dual to the given base. We deduce that either $\lambda$ appears in Table 11 or in Table 2 or $\lambda=\omega_{1}+\omega_{3}, 2 \omega_{3}$, $(p-3) \omega_{1}+2 \omega_{3}$, or $(p-2) \omega_{1}+\omega_{3}$. The first case is ruled out by Lemma 3.2(4). For the second and third, where $p>2$, we see that the restriction of $\lambda$ to the standard $C_{2}$ Levi factor affords a composition factor isomorphic to the Lie algebra of the Levi factor, in which the non-zero weight $\lambda-\alpha_{2}-2 \alpha_{3}$ has multiplicity 2 . For the final case, when $\lambda=(p-2) \omega_{1}+\omega_{3}$, consider the $C_{2}$ subsystem subgroup $X^{s_{1}}$, with root system base $\left\{\alpha_{1}+\alpha_{2}, \alpha_{3}\right\}$, for which $\lambda$ affords a composition factor with highest weight $(p-2) \zeta_{1}+\zeta_{2}$ (where $\zeta_{1}, \zeta_{2}$ are the fundamental dominant weights with respect to the base $\left.\left\{\alpha_{1}+\alpha_{2}, \alpha_{3}\right\}\right)$, contradicting Lemma 3.3. This completes the consideration of $G=B_{3}$.

Consider now the general case $n \geq 4$. Let $\lambda=\sum a_{i} \omega_{i}$. By considering the restriction of $\lambda$ to the standard $B_{3}$ Levi subgroup, we see that $a_{n-1}+a_{n}<p$. Now consider the maximal rank $D_{n}$ subsystem subgroup $W$ with root system base $\left\{\alpha_{1}, \ldots, \alpha_{n-2}, \alpha_{n-1}, \alpha_{n-1}+2 \alpha_{n}\right\}$. The above remarks imply that $\left.\lambda\right|_{T \cap X}$ is a $p$-restricted weight and the result for $D_{n}$ then gives the result for $B_{n}$.

Case $\mathbf{E}_{\mathbf{n}}$. For $n=6$, we apply the $D_{n}$ result to the two standard $D_{5}$ Levi subgroups of $G$ and the $A_{n}$ result to the standard $A_{5}$ Levi subgroup of $G$ to see that either $\lambda$ is as in Table 2 or $\lambda=\omega_{3}, \omega_{5}, 2 \omega_{1}$ or $2 \omega_{6}$. In the first two cases, the restriction of $\lambda$ to one of the $D_{5}$ Levi factors affords a composition factor which is isomorphic to the unique nontrivial composition factor of the Lie algebra of the Levi factor. But the zero weight in this composition factor corresponds to a non-zero weight with multiplicity at least 4 . In the last two configurations, we note that the $D_{5}$ composition factor afforded by $\lambda$ has zero as a subdominant weight of multiplicity at least 3 (here we use [Lüb]). But this subdominant weight is a non-zero weight with respect to $T$.

Now for $n=7$, we apply the result for $E_{6}$ as well as for $D_{6}$ and $A_{6}$ to see 
that either $\lambda$ is as in the statement of the result or $\lambda=\omega_{6}$ or $2 \omega_{7}$. These two configurations can be ruled out exactly as in the case of $E_{6}$. The case of $G=E_{8}$ is completely analogous.

Case $\mathbf{F}_{4}$. For this case, we use the standard $B_{3}$ and $C_{3}$ Levi factors and the maximal rank $D_{4}$ subsystem subgroup whose root system base is $\left\{\alpha_{2}+2 \alpha_{3}+\right.$ $\left.2 \alpha_{4}, \alpha_{2}, \alpha_{1}, \alpha_{2}+2 \alpha_{3}\right\}$. This leads immediately to the result.

Case $\mathbf{G}_{\mathbf{2}}$. Let $\lambda=b \omega_{1}+a \omega_{2}$, where $3\left(\alpha_{1}, \alpha_{1}\right)=\left(\alpha_{2}, \alpha_{2}\right)$. We first treat the cases where $p=2$ or $p=3$ by referring to Lüb to see that $\lambda$ is as in Table 2 or $\lambda=2 \omega_{1}+2 \omega_{2}$ and $p=3$. But then Lemma 3.2(5) shows that the non-zero weight $\lambda-\alpha_{1}-\alpha_{2}$ has multiplicity 2 . So we may now assume $p>3$. We will consider the restriction of $\lambda$ to $X$, the $A_{2}$ subsystem subgroup corresponding to the long roots in $\Phi(G)$; we have $\left.\lambda\right|_{T \cap X}=(a+b) \eta_{1}+a \eta_{2}$, where $\left\{\eta_{1}, \eta_{2}\right\}$ are the fundamental dominant weights for $X$.

Assume that $\lambda$ is not as in Table 2, so $\lambda \neq \omega_{i}, i=1,2$. Now Lemma3.2(8) implies that $a \neq 0$. Consideration of the action of $X$, together with Lemma 3.2(1), implies that either $a+b \geq p$ or $2 a+b+1 \equiv 0(\bmod p)$. Now if $b=0$, we must be in the second case, so $a=\frac{p-1}{2}$; in particular, $a>1$. But then Lemma $3.2(9)$ gives a contradiction. Hence we must have $b \neq 0$. Then Lemma $3.2(5)$ implies that $3 a+b+3 \equiv 0(\bmod p)$ and either $2 a+b+1 \equiv 0(\bmod p)$ or $a+b \geq p$. In the first case we have $a=p-2$ and $b=3$; in the second case we must have $b \geq 2$. So in either case $\lambda-2 \alpha_{1}-\alpha_{2}$ is a subdominant weight. Arguing as in [Sei87, 6.18], we see that the non-zero weight $\lambda-2 \alpha_{1}-\alpha_{2}$ has multiplicity 1 only if $6 a+4 b+8 \equiv 0(\bmod p)$, which together with the previous congruence relation implies that $b=p-1$, and hence $3 a+2 \equiv 0(\bmod p)$. We are now in the situation where $a+b \geq p$; indeed, as $a=\frac{p-2}{3}$ or $\frac{2 p-2}{3}$, we have that either $p=5, a=1, b=4$ and [Lüb] gives a contradiction, or $\left.\lambda\right|_{T \cap X}=p \eta_{1}+\left(c \eta_{1}+d \eta_{2}\right)$, with $c d \neq 0,(c, d)=\left(\frac{p-5}{3}, \frac{p-2}{3}\right)$ or $\left(\frac{2 p-5}{3}, \frac{2 p-2}{3}\right)$. In each case, the result for $A_{2}$ leads to a contradiction.

It remains to verify for each weight $\lambda$ in Table 2 that all non-zero weights of $L_{G}(\lambda)$ do indeed have multiplicity 1 . This is straightfoward using [Lüb01] and Lüb].

The following corollary is immediate.

Corollary 3.5. Let $\lambda \in X(T)$. If all non-zero weights of $L_{G}(\lambda)$ occur with multiplicity 1 , then the zero weight occurs with multilicity at most $\operatorname{rank}(G)$.

Note that lower bounds for the maximal weight multiplicities in irreducible representations of $G$ are studied in [BOS08, BOS14]. The above corollary does not however follow from their results.

Corollary 3.6. Let $\lambda \in \Omega_{2}(G)$. Then one of the following holds:

(1) $\lambda \in \Omega_{1}(G)$.

(2) $L_{G}(\lambda)$ is the unique nontrivial composition factor of $\operatorname{Lie}(G)$. 


\begin{tabular}{|l|c|c|c|}
\hline$G$ & $\lambda$ & conditions & $\operatorname{dim} L_{G}(\lambda)$ \\
\hline$A_{2}$ & $\omega_{1}+\omega_{2}$ & $p \neq 3$ & 8 \\
\hline$A_{3}$ & $2 \omega_{2}$ & $p>3$ & 20 \\
& $\omega_{1}+\omega_{3}$ & $p=2$ & 14 \\
\hline$C_{2}$ & $2 \omega_{1}$ & & 10 \\
& $2 \omega_{2}$ & $p \neq 5$ & 14 \\
\hline$C_{3}$ & $\omega_{2}$ & $p \neq 3$ & 14 \\
\hline$C_{4}$ & $\omega_{2}$ & $p=2$ & 26 \\
& $\omega_{4}$ & $p \neq 2,3$ & 42 \\
\hline$D_{4}$ & $\omega_{2}$ & $p=2$ & 26 \\
\hline$F_{4}$ & $\omega_{1}$ & $p=2$ & 26 \\
& $\omega_{4}$ & $p \neq 3$ & 26 \\
\hline$G_{2}$ & $\omega_{2}$ & $p \neq 3$ & 14 \\
\hline
\end{tabular}

Table 3: $\lambda \in \Omega_{2}(G), 0$ weight in $L_{G}(\lambda)$ of multiplicity 2

(3) $(G, \lambda)$ is one of $\left(A_{3}, 2 \omega_{2}\right),\left(B_{n}, 2 \omega_{1}\right),\left(C_{n}, \omega_{2}\right),\left(C_{2}, 2 \omega_{2}\right),\left(C_{4}, \omega_{4}\right),\left(D_{n}, 2 \omega_{1}\right)$, $\left(F_{4}, \omega_{4}\right)$, where $p \neq 3$ in the latter case.

In addition, the zero weight multiplicity in cases (2), (3) is as in Table 2.

Proof. The zero weight multiplicity can be easily computed from the knowledge of the dimension of $L_{G}(\lambda)$, provided in [Lüb01]. The statement about $\operatorname{Lie}(G)$ follows from the known structure of the $F G$-module $\operatorname{Lie}(G)$; see for example [Sei87, 1.9].

We record in the following corollary the cases where the 0 weight has multiplicity 2 in $L_{G}(\lambda)$, for $\lambda \in \Omega_{2}(G)$. This will be required for the resolution of Problem 1, in case $H=D_{n}$.

Corollary 3.7. Let $\lambda \in \Omega_{2}(G)$ and suppose that the zero weight has multiplicity 2 in $L_{G}(\lambda)$. Then the pair $(G, \lambda)$ is as in Table 3 .

We can now complete the proof of Theorem 1.2

Theorem 3.8. Let $\lambda \in X(T)$ be a non-zero dominant weight. Then at most one weight space of $L_{G}(\lambda)$ is of dimension greater than one if and only if one of the following holds:

(1) the module $L_{G}(\lambda)$ is as described in Proposition 3.1;

(2) $\lambda=p^{k} \mu$ for some $k \in \mathbb{N}, k=0$ if $\operatorname{char}(F)=0$, and for some weight $\mu \in \Omega_{2}(G) \backslash \Omega_{1}(G)$, as given in Table 2 .

Proof. By Propositions 3.1 and 3.4, the modules $L_{G}(\lambda)$ described in (1) and (2), all non-zero weights are of multiplicity at most 1 . So now take $\lambda \in X(T)$ a non-zero dominant weight such that at most one weight space of $L_{G}(\lambda)$ has 
dimension greater than 1 , and suppose $\lambda$ is not as in (1). In particular, $L_{G}(\lambda)$ has a weight of multiplicity greater than 1 . Note that if a non-zero weight in $L_{G}(\lambda)$ occurs with multiplicity greater than 1 , then so do all of its conjugates under the Weyl group. Hence, the weight occurring with multiplicity greater than one in $L_{G}(\lambda)$ must be the zero weight.

Now, let $\lambda=\sum_{i=1}^{l} p^{k_{i}} \lambda_{i}$, where $\lambda_{i}$ is a non-zero dominant $p$-restricted weight for all $i$; so we have $L_{G}(\lambda) \cong L_{G}\left(p^{k_{1}} \lambda_{1}\right) \otimes \cdots \otimes L_{G}\left(p^{k_{l}} \lambda_{l}\right)$. If $l=1$ then (2) holds. Let $l>1$. Then for all $1 \leq i \leq l$, the weights of $L_{G}\left(p^{k_{i}} \lambda_{i}\right)$ must have multiplicity 1 (else a non-zero weight has multiplicity greater than 1 in $L_{G}(\lambda)$ ). Then by Proposition 3.1, we see that there exists a pair $\left(\lambda_{i}, \lambda_{j}\right)$ with $k_{j}=k_{i}+1$, as in Proposition 3.1(2)(b). So we investigate the multiplicity of the zero weight in the 2-fold tensor products associated to these pairs of weights.

Let $L_{G}(\lambda)=L_{G}\left(\lambda_{1}\right) \otimes L_{G}\left(p \lambda_{2}\right)$, where $\left(G, p, \lambda_{1}, \lambda_{2}\right)$ are the following: (a) $\left(C_{n}, 2, \omega_{n}, \omega_{1}\right)$, (b) $\left(G_{2}, 2, \omega_{1}, \omega_{1}\right)$, (c) $\left(G_{2}, 3, \omega_{2}, \omega_{1}\right)$.

In case (a), let $\varepsilon_{i}$ be the weights defined as in [Bou68, Planche III, IX]. The weights of $L_{G}\left(\omega_{n}\right)$ are $\pm \varepsilon_{1} \pm \cdots \pm \varepsilon_{n}$ and the weights of $L_{G}\left(2 \omega_{1}\right)$ are $\pm 2 \varepsilon_{1}, \ldots, \pm 2 \varepsilon_{n}$; in particular, there are no common weights. It follows that the 0 weight does not occur in $L_{G}\left(\lambda_{1}\right) \otimes L_{G}\left(2 \lambda_{2}\right)$.

In case (b), the weights of $L_{G}\left(\omega_{1}\right)$ are $\left\{ \pm\left(\varepsilon_{1}-\varepsilon_{2}\right), \pm\left(\varepsilon_{1}-\varepsilon_{3}\right), \pm\left(\varepsilon_{2}-\varepsilon_{3}\right)\right\}$. So $L_{G}\left(\omega_{1}\right)$ and $L_{G}\left(2 \omega_{1}\right)$ again have no common weight, and hence the 0 weight does not occur in $L_{G}\left(\omega_{1}\right) \otimes L_{G}\left(2 \omega_{1}\right)$.

Finally for case (c), the weights of $L_{G}\left(3 \omega_{1}\right)$ are $\left\{0,\left\{ \pm 3\left(\varepsilon_{1}-\varepsilon_{2}\right), \pm 3\left(\varepsilon_{1}-\right.\right.\right.$ $\left.\left.\varepsilon_{3}\right), \pm 3\left(\varepsilon_{2}-\varepsilon_{3}\right)\right\}$, and the weights of $L_{G}\left(\omega_{2}\right)$ are $0, \pm\left(2 \varepsilon_{1}-\varepsilon_{2}-\varepsilon_{3}\right), \pm\left(2 \varepsilon_{3}-\varepsilon_{1}-\right.$ $\left.\varepsilon_{2}\right), \pm\left(2 \varepsilon_{2}-\varepsilon_{1}-\varepsilon_{3}\right)$. Then the multiplicity of the weight 0 in $L_{G}\left(\lambda_{1}\right) \otimes L_{G}\left(3 \lambda_{2}\right)$ is 1 .

It follows from Proposition 3.1 that the weights of $L_{G}\left(\lambda_{1}\right) \otimes L_{G}\left(p \lambda_{2}\right)$ whose multiplicity is greater than 1 are non-zero. Hence there are no examples of $\lambda=\sum_{i=1}^{l} p^{k_{i}} \lambda_{i}$ with $l>1$ and all $\lambda_{i}$ different from 0 such that the zero weight has multiplicity greater than 1 and all non-zero weights have multiplicity 1 in the module $L_{G}(\lambda)$.

\section{Semisimple regular elements in classical groups}

Throughout this section, $H$ is a simply connected simple algebraic group of classical type, with natural module $V$. Let $\tilde{H}$ be the image of $H$ in $\operatorname{GL}(V)$. Note that $x \in H$ is regular if and only if the image of $x$ in $\tilde{H}$ is regular in $\tilde{H}$. Moreover, we will assume $p>2$ when $H$ is of type $B_{m}$, as in the case $p=2$, it will suffice to treat the group of type $C_{m}$.

Lemma 4.1. Let $H$ be of type $B_{m}, C_{m}$ or $D_{m}$. Let $t \in \tilde{H}$ be a semisimple regular element. Then $t$ is a regular element in $\mathrm{GL}(V)$, except for the following cases:

(1) $H$ is of type $B_{m}$ and -1 is an eigenvalue of $t$ on $V$ with multiplicity 2; 
(2) $H$ is of type $D_{m}$ and either 1 or -1 or both are eigenvalues of $t$ on $V$ with multiplicity 2.

Proof. Let $d=\operatorname{dim} V$ and let $b_{1}, \ldots, b_{d}$ be a basis in $V$ with respect to which the Gram matrix of the bilinear form is anti-diagonal, with all non-zero entries in the set $\{1,-1\}$. We may assume the matrix of $t$ with respect to this basis is diagonal and of the form $t=\operatorname{diag}\left(t_{1}, \ldots, t_{m}, x, t_{m}^{-1}, \ldots, t_{1}^{-1}\right)$, where $x$ is absent if $d$ is even and equal to 1 otherwise. Since $t$ is regular in $H, t_{1}, \ldots, t_{m}$ are distinct. Moreover, if $H$ is of type $C_{m}$ then all $t_{1}, \ldots, t_{m}, t_{m}^{-1}, \ldots, t_{1}^{-1}$ are distinct. Indeed, the roots of $C_{m}$ take values $\left\{t_{i} t_{j}^{-1}, t_{i} t_{j}, t_{j}^{2} \mid 1 \leq i \neq j \leq m\right\}$ and $t$ lies in the kernel of no root. If $H$ is of type $B_{m}, m>2$, then the roots of $H$ take values $\left\{t_{i} t_{j}^{-1}, t_{i} t_{j}, t_{j} \mid 1 \leq i \neq j \leq m\right\}$ on $t$. So no $t_{i}$ is equal to 1 and therefore the eigenvalues $t_{1}, \ldots, t_{m}, x, t_{m}^{-1}, \ldots, t_{1}^{-1}$ are distinct except for the case (1). Finally, for $H$ of type $D_{m}$, we argue as above, using the fact that the roots of $H$ take values $\left\{t_{i} t_{j}^{-1}, t_{i} t_{j} \mid 1 \leq i \neq j \leq m\right\}$ on $t$, which leads to (2).

Lemma 4.2. Let $H$ be of type $B_{m}, C_{m}$ or $D_{m}$, and let $T^{\prime}$ be a regular torus in $\tilde{H}$. Then $T^{\prime}$ is a regular torus in $\mathrm{GL}(V)$, except for the case where $H$ is of type $D_{m}$ and the fixed point subspace of $T^{\prime}$ on $V$ is of dimension 2.

Proof. Appying Lemma 1.7, we choose $t \in T^{\prime}$ such that $C_{H}(t)^{\circ}=C_{H}\left(T^{\prime}\right)$. By Lemma 4.1, $t$ is regular in $\mathrm{GL}(V)$ if $H$ is of type $C_{m}$, and so the result holds. If $\operatorname{dim} V$ is odd, that is, if $H$ is of type $B_{m}$, let $T$ be a maximal torus of $H$. Then for all $T$-weights $\mu, \nu$ of $V$, the difference $\mu-\nu$ is a multiple of a root of $H$ (with respect to $T$ ). Since $T^{\prime}$ is a regular torus in $\tilde{H}, T^{\prime} \not \subset \operatorname{ker}(\beta)$ for any $\operatorname{root} \beta$ of $H$. Hence $T^{\prime}$ has distinct weights on $V$, and so is a regular torus in GL $(V)$.

Finally, consider the case $H=D_{m}$. Let $b_{1}, \ldots, b_{2 m}$ be a basis for $V$ as in the previous proof. We may assume that $T^{\prime}$ consists of diagonal matrices with respect to this basis. Now, $T^{\prime}$ regular implies that the weight of $T^{\prime}$ afforded by $\left\langle b_{j}\right\rangle$ is distinct from the weight afforded by $\left\langle b_{2 m-k+1}\right\rangle$ for all $k \notin\{j, 2 m-j+1\}$. So the weights of $T^{\prime}$ on $V$ are distinct unless there exists $j, 1 \leq j \leq m$, such that the weight afforded by $\left\langle b_{j}\right\rangle$ is equal to that afforded by $\left\langle b_{2 m-j+1}\right\rangle$. So assume these two weights coincide. Since the weights afforded by these two vectors differ by a sign, if they are equal, they must be 0 . Hence there is a unique such $j$ and the result holds.

Proposition 4.3. Let $H$ be of type $D_{m}$ with $m \geq 4$. Let $T$ be a torus in $\tilde{H}$. Suppose that there exists at most one $T$-weight of $V$ of multiplicity greater than 1 , and if such a weight exists, its multiplicity is 2 . Then $C_{\tilde{H}}(T)$ is a maximal torus in $\tilde{H}$, that is, $T$ is a regular torus in $\tilde{H}$.

Proof. If all weights of $T$ on $V$ have multiplicity 1 , then $T$ is a regular torus in $\operatorname{GL}(V)$, and hence in $\tilde{H}$. Suppose that there is a weight $\mu$ of $T$ on $V$ of multiplicity 2 , and let $M$ be the corresponding weight space. Denote by $R$ the set of singular vectors in $M$ together with the zero vector.

If $R=M$ then $M$ is totally singular, $M \subset M^{\perp}$, and $\left.V\right|_{T}=M^{\perp} \oplus V_{2}$. It is well-known that the $F T$-module $V_{2}$ is dual to $M$, and hence is contained in a 
$T$-weight space. This is a contradiction as $\operatorname{dim} V_{2}=2$ and by hypothesis $M$ is the unique weight space of dimension greater than 1 .

Suppose that $M$ is neither totally singular nor non-degenerate with respect to the bilinear form on $V$. Then $R$ is a 1-dimensional $T$-invariant subspace of $M$ and $M \subset R^{\perp}$. Let $\left.M\right|_{T}=R \oplus R^{\prime}$ for a $T$-submodule $R^{\prime}$. Then non-zero vectors $x \in R^{\prime}$ are non-singular, and if $t \in T$ then $t x=\mu(t) x$. Let $Q$ be the quadratic form on $V$ preserved by $\tilde{H}$. Then $Q(x)=Q(t x)=\mu(t)^{2} Q(x)$, for all $t \in T$, whence $\mu=0$. Therefore, $T$ acts trivially on $M$, and hence on $R$. However, $V / R^{\perp}$ is an $F T$-module dual to $R$, so $T$ acts trivially on $V / R^{\perp}$. As $V$ is a completely reducible $F T$-module, it follows that the dimension of the zero $T$-weight space on $V$ is at least 3, which contradicts the hypothesis.

Finally, suppose that $M$ is non-degenerate with respect to the bilinear form on $V$. The reductive group $C_{\tilde{H}}(T)$ stabilizes all $T$-weight spaces of $V$. Since $\mathrm{SO}_{2}(F)$ has no unipotent elements, $C_{\tilde{H}}(T)$ must be a torus, as required.

Proof of Proposition 1.1. The Proposition follows directly from Lemma 4.2 and Proposition 4.3.

As explained in Section 1 and Section 2, for a classical group $H$ with natural module $V$, Proposition 1.1 reduces the resolution of Problems 1 and 2 to a question about weight multiplicities in the module $\left.V\right|_{G}$, for a simple algebraic group $G$ acting irreducibly and tensor-indecomposably on $V$. Given a simple subgroup $G$ of $H$, we see that a maximal torus $T$ of $G$ is a regular torus of $H$ if and only if one of the following holds:

a) all $T$-weight spaces of $V$ are 1-dimensional, and so $V$ is described by Proposition 3.1, or

b) $G \subset H=D_{m}$ and the 0 weight space of $V$ (viewed as a $T$-module) is 2dimensional, while all other $T$-weight spaces of $V$ are 1-dimensional, so $V$ is described by Theorem 3.8 and Proposition 3.4 .

\section{Orthogonal and symplectic representations}

As in the previous sections, we take $G, T$ and the rest of the notation to be as fixed in Section 1. In this section we partition the irreducible representations $\rho$ of $G$ with highest weight in $\Omega_{1}(G)$ into four families depending on whether $\rho(G)$ is contained in a group of type $B_{m}, C_{m}, D_{m}$ or in none of them. (It is well-known that the latter holds if and only if the associated $F G$-module is not self-dual.) In addition, we determine which of the weights in $\Omega_{2}(G)$ correspond to a representation whose image contains a regular torus of $H=D_{m}$. This information is collected in Tables 4, 5, 6, 7, 8, 9, and completes the proof of Theorem 1.5. For simplicity, we will say that an irreducible representation of $G$ (or the corresponding module $V$ ) is symplectic, respectively orthogonal, if $G$ preserves a non-degenerate alternating form, respectively a non-degenerate 
quadratic form on $V$. As discussed in Section 2, for our application, it suffices to consider tensor-indecomposable modules.

Until the end of the section $\lambda \in X(T)$ is assumed to be a $p$-restricted dominant weight. Recall that the highest weight of the irreducible $F G$-module $L_{G}(\lambda)^{*}$ (the dual of $L_{G}(\lambda)$ ), is $-w_{0} \lambda$, where $w_{0}$ is the longest word in the Weyl group of $G$. Since $-w_{0}=$ id for the groups $A_{1}, B_{n}, C_{n}, D_{n}$, ( $n$ even), $E_{7}, E_{8}, F_{4}$, and $G_{2}$, all irreducible modules are self-dual for these groups. (See [MT11, 16.1].) The following result treats the remaining cases.

Proposition 5.1. Let $G$ be of type $A_{n}, n>1, D_{n}, n$ odd, or $E_{6}$. Let $\lambda \in \Omega_{2}(G)$. Then $L_{G}(\lambda)$ is a self-dual FG-module if and only if one of the following holds:

(1) $G=A_{n}, n>1$, and either

(i) $\lambda=\omega_{1}+\omega_{n}$, or

(ii) $\lambda=\omega_{(n+1) / 2}$, for $n$ odd, or

(iii) $\lambda=(p-1) \omega_{(n+1) / 2}$, for $n$ odd, or

(iv) $\lambda=\frac{p-1}{2}\left(\omega_{n / 2}+\omega_{(n+2) / 2}\right)$, for $n$ even and $p$ odd, or

(v) $\lambda=2 \omega_{2}$ for $n=3$.

(2) $G \cong D_{n}, n$ odd, and $\lambda \in\left\{\omega_{1}, \omega_{2}, 2 \omega_{1}\right\}$;

(3) $G \cong E_{6}$ and $\lambda=\omega_{2}$.

Proof. The above remarks imply that $L_{G}(\lambda)$ is self-dual if and only if $\lambda=-w_{0} \lambda$, that is, $\lambda$ is invariant under the graph automorphism of $G$. We refer to Table 2 for the weights $\lambda \in \Omega_{2}(G)$. The cases where $G$ is of type $E_{6}$ or $D_{n}$ are trivial. For $G=A_{n}, n>1$, consider $\omega=c \omega_{i}+(p-1-c) \omega_{i+1}$, where $0 \leq c<p$. The graph automorphism sends $\omega_{j}$ to $\omega_{n-j+1}$. If $n$ is even, it follows that $i+1=n-i+1$ and $c=p-1-c$. So $i=n / 2$ and $c=(p-1) / 2$. Similarly, for $n$ odd we get $\omega=(p-1) \omega_{(n+1) / 2}$.

Lemma 5.2. (1) Let $G=A_{n}, p>2$ and let $\lambda=(p-1) \omega_{(n+1) / 2}$ for $n$ odd, and $\lambda=\frac{p-1}{2}\left(\omega_{n / 2}+\omega_{(n+2) / 2}\right)$ for $n$ even. Then $\operatorname{dim} L_{G}(\lambda)$ is odd.

(2) Let $G=A_{n}, n$ odd, and $\lambda=\omega_{(n+1) / 2}$. Then $\operatorname{dim} L_{G}(\lambda)=\left(\begin{array}{c}n+1 \\ (n+1) / 2\end{array}\right)$ and so $\operatorname{dim} L_{G}(\lambda)$ is even.

(3) Let $G=C_{n}, n>1, p>2$ and let $\lambda_{1}=\frac{p-1}{2} \omega_{n}, \lambda_{2}=\omega_{n-1}+\frac{p-3}{2} \omega_{n}$. Then $\operatorname{dim} L_{G}\left(\lambda_{1}\right)=\left(p^{n}+1\right) / 2$ and $\operatorname{dim} L_{G}\left(\lambda_{2}\right)=\left(p^{n}-1\right) / 2$.

Proof. (1) Let $B:=\{(c, i) \mid 0 \leq c \leq p-1,0 \leq i \leq n\}$ and for the purposes of this proof set $\omega_{0}$ and $\omega_{n+1}$ to be the 0 weight. Then by [ZS90, Prop. 1.2] (and the discussion on page 555 loc.cit), the direct sum of all irreducible representations $L_{G}(\mu)$ with $\mu$ running over the set $\left\{(p-1-c) \omega_{i}+c \omega_{i+1} \mid(c, i) \in B\right\}$ has dimension $p^{n+1}$. Note that the trivial representation of $G$ occurs twice among the $L_{G}(\mu)$ 
(specifically, for $(c, i)=(0,0)$ and $(p-1, n))$. We also observe that $L_{G}(\lambda)$ with $\lambda$ as in (1) is the only nontrivial self-dual module among the $L_{G}(\mu)$, whereas the other $L_{G}(\mu)$ (with $\mu \neq \lambda, 0$ ) occur in the sum as dual pairs. Therefore, the parity of $\operatorname{dim} L_{G}(\lambda)$ coincides with that of $p^{n+1}$, which is an odd number.

(2) The irreducible $F G$-module $L_{G}\left(\omega_{j}\right)$ is the $j$-th exterior power of the natural $F G$-module, whence the result.

(3) The assertion is proven in [ZS87, see the statement A of the Main Theorem.

Proposition 5.3. Let $\lambda \in \Omega_{2}(G)$. Assume moreover that $p>2$. Then $L_{G}(\lambda)$ is symplectic if and only if the pair $(G, \lambda)$ is as in Table 4 . In particular, if $\lambda \in \Omega_{2}(G)$ with $L_{G}(\lambda)$ symplectic, then $\lambda \in \Omega_{1}(G)$.

Proof. First, by [Ste68, Lemma 79], if $|Z(G)|$ is odd then every self-dual irreducible representation of $G$ is orthogonal; in particular, this is the case if $G=A_{n}$ with $n$ even. So we assume that $|Z(G)|$ is even. It follows that $G$ is classical or of type $E_{7}$. Furthermore, in the adjoint representation of $G$ the center acts trivially, so again by [Ste68, Lemma 79] the representations arising from the adjoint representation are orthogonal. More generally, any representation where all weights are roots must be orthogonal. Now let $h \in Z(G)$ as defined in [Ste68, Lemma 79].

Using the description of the sum of the positive roots in $\Phi\left(E_{7}\right)$ given in [Bou68, Planche VI], we deduce that $h$ acts nontrivially on $L_{E_{7}}\left(\omega_{7}\right)$, and hence by [Ste68, Lemma 79] $L_{E_{7}}\left(\omega_{7}\right)$ is symplectic. So we are left with the classical groups.

Let $G=A_{n}$ with $n$ odd. One checks that $h$ is the central involution in $G$. In view of Proposition 5.1, Lemma 5.2, and the above comments, we must consider the cases $\lambda=2 \omega_{2}$ for $n=3$ and $\lambda=\omega_{(n+1) / 2}$ with $n$ odd. In the former case, $h$ acts trivially on $L_{G}\left(2 \omega_{2}\right)$, so the module is orthogonal. In the second case, $L_{G}(\lambda)$ is the $(n+1) / 2$-th exterior power of the natural $F G$-module, and so $h$ acts as $(-1)^{(n+1) / 2} \cdot \operatorname{Id}$ on $L_{G}(\lambda)$. Then [Ste68, Lemma 79] gives the result.

Let $G=C_{n}, n>1$. Since $L_{G}\left(\omega_{1}\right)$ is the natural symplectic module for $G, h$ acts as $-\operatorname{Id}$ on $L_{G}\left(\omega_{1}\right)$. As $Z(G)$ is of order $2, L_{G}(\lambda)$ is symplectic if and only if $G$ acts faithfully on $L_{G}(\lambda)$. As the root lattice $\mathbb{Z} \Phi(G)$ has index 2 in the weight lattice, it follows that $G$ acts faithfully on $L_{G}(\lambda)$ if and only if $\lambda \notin \mathbb{Z} \Phi(G)$. The weights $\lambda=\omega_{2}, 2 \omega_{1}, 2 \omega_{2}$ all lie in $\mathbb{Z} \Phi(G)$. Note that $\omega_{n}$ (respectively, $\omega_{n-1}$ ) lies in $\mathbb{Z} \Phi(G)$ if and only if $n$ is even (respectively, odd). (See [Bou68, ].) Therefore, $\frac{p-1}{2} \omega_{n} \notin \mathbb{Z} \Phi(G)$ if and only if $\frac{n(p-1)}{2}$ is odd. Observe that $\omega_{n}-\omega_{n-1} \notin \mathbb{Z} \Phi(G)$ and $\omega_{n-1}+\frac{p-3}{2} \omega_{n}=\omega_{n-1}-\omega_{n}+\frac{p-1}{2} \omega_{n} ;$ it follows that $\omega_{n-1}+\frac{p-3}{2} \omega_{n} \notin \mathbb{Z} \Phi(G)$ if and only if $\frac{n(p-1)}{2}$ is even, as stated in Table 4 .

Let $G=D_{n}, n>3$ odd; here the weights which we must consider are $\omega_{1}, 2 \omega_{1}$ and $\omega_{2}$. Since $L_{G}\left(\omega_{1}\right)$ is the natural orthogonal module for $G, h$ acts trivially on $L_{G}\left(\omega_{1}\right)$. Since $2 \omega_{1}$ and $\omega_{2}$ each occur in $L_{G}\left(\omega_{1}\right) \otimes L_{G}\left(\omega_{1}\right), L_{G}\left(2 \omega_{1}\right)$ and $L_{G}\left(\omega_{2}\right)$ are also orthogonal. 


\begin{tabular}{|l|c|c|c|}
\hline$G$ & $\lambda$ & conditions & $\operatorname{dim} L_{G}(\lambda)$ \\
\hline$A_{1}$ & $a \omega_{1}$ & $a$ odd & $a+1$ \\
\hline & & $n>1$ odd $\frac{n+1}{2}$ odd & $\left(\begin{array}{c}n+1 \\
\frac{n+1}{2}\end{array}\right)$ \\
$A_{n}$ & $\omega_{(n+1) / 2}$ & $n>1$ & $2^{n}$ \\
\hline$B_{n}$ & $\omega_{n}$ & $n>2, n \equiv 1$ or $2 \quad(\bmod 4)$ & $2 n$ \\
\hline$C_{3}$ & $\omega_{3}$ & $n$ & $\left(p^{n}-1\right) / 2$ \\
$C_{n}$ & $\omega_{1}$ & $\left(p^{n}+1\right) / 2$ \\
& $\omega_{n-1}+\frac{p-3}{2} \omega_{n}$ & $n>1, p \geq 3, n(p-1) / 2$ even \\
& $\frac{p-1}{2} \omega_{n}$ & $n>1, p \geq 3, n(p-1) / 2$ odd & $2^{n-1}$ \\
\hline$D_{n}$ & $\omega_{n-1}, \omega_{n}$ & $n>3$ even, $n \equiv 2 \quad(\bmod 4)$ & 56 \\
\hline$E_{7}$ & $\omega_{7}$ & &
\end{tabular}

Table 4: $\lambda \in \Omega_{2}(G), p \neq 2, L_{G}(\lambda)$ symplectic

Let $G=D_{n}, n>3$ even. For the weights $\omega_{1}, 2 \omega_{1}$ and $\omega_{2}$, the argument of the previous paragraph is valid. So we must consider the weights $\omega_{n-1}$ and $\omega_{n}$. When $n=4$, a direct check using the information in [Bou68, Planche IV] allows one to see that $\omega_{n-1}(h)=(-1)^{\frac{n(n-1)}{2}}=\omega_{n}(h)$, and so $L_{G}\left(\omega_{n-1}\right)$ and $L_{G}\left(\omega_{n}\right)$ are symplectic if and only if $n \equiv 2(\bmod 4)$.

Let $G=B_{n}, n>2$. In this case $\omega_{n}$ is the only fundamental dominant weight which does not lie in the root lattice. In the natural embedding of $B_{n}$ in $D_{n+1}$, $B_{n}$ acts irreducibly on each of the spin modules for $D_{n+1}$; the restriction is the $F G$-module $L_{B_{n}}\left(\omega_{n}\right)$ in each case. Hence, for $n$ odd, $L_{G}\left(\omega_{n}\right)$ is symplectic if and only if $n+1 \equiv 2(\bmod 4)$. When $n$ is even, we consider the natural embedding of $D_{n}$ in $B_{n}$, where the spin module for $B_{n}$ decomposes as a direct sum of the two distinct spin modules for $D_{n}$, and the summands are non-degenerate with respect to the form. Hence $L_{G}\left(\omega_{n}\right)$ is symplectic if and only if $n \equiv 2(\bmod 4)$. So to summarize, for the group $G=B_{n}, L_{G}\left(\omega_{n}\right)$ is symplectic if and only if $n \equiv 1$ or $2(\bmod 4)$.

This completes the proof of the proposition.

Continuing with the case where $G$ preserves a non-degenerate form on $L_{G}(\lambda)$, for the weights $\lambda \in \Omega_{2}(G)$ not listed in Table 4 , the module $L_{G}(\lambda)$ is orthogonal. In order to decide whether the image of $G$ under the corresponding representation belongs to a subgroup of type $B_{n}$ or $D_{n}$, one has only to determine whether $\operatorname{dim} L_{G}(\lambda)$ is odd or even. Since we are interested in the solution to Problem 2, we consider those weights $\lambda \in \Omega_{2}(G)$ for which the multiplicity of the 0 weight is at most 1 , if $L_{G}(\lambda)$ is odd-dimensional, and at most 2 if $\operatorname{dim} L_{G}(\lambda)$ is even.

The following lemma can be deduced directly from Lü̈01] and the preceding 


\begin{tabular}{|l|c|c|c|}
\hline$G$ & $\lambda$ & conditions & $\operatorname{dim} L_{G}(\lambda)$ \\
\hline & & & \\
$A_{n}$ & $\omega_{(n+1) / 2}$ & $n>1$ odd, $(n+1) / 2$ even & $\left(\begin{array}{c}n+1 \\
\frac{n+1}{2}\end{array}\right)$ \\
& & $p \neq 3$ & 8 \\
$A_{2}$ & $\omega_{1}+\omega_{2}$ & $p>3$ & 20 \\
$A_{3}$ & $2 \omega_{2}$ & $n \equiv 0$ or $3 \quad(\bmod 4)$ & $2^{n}$ \\
\hline$B_{n}$ & $\omega_{n}$ & $p \neq 5$ & 10 \\
\hline$C_{2}$ & $2 \omega_{1}$ & 14 \\
& $2 \omega_{2}$ & $p>3$ & 14 \\
$C_{3}$ & $\omega_{2}$ & $n>3$ & 42 \\
$C_{4}$ & $\omega_{4}$ & $n \equiv 0 \quad(\bmod 4)$ & $2^{n-1}$ \\
\hline$D_{n}$ & $\omega_{1}$ & $p>3$ & 26 \\
\hline$F_{4}$ & $\omega_{n-1}, \omega_{n}$ & $n>3$ & 14 \\
\hline$G_{2}$ & $\omega_{2}$ & $p$ &
\end{tabular}

Table 5: $\lambda \in \Omega_{2}(G), \quad p \neq 2, L_{G}(\lambda)$ even-dimensional orthogonal, with 0 weight of multiplicity at most 2

results.

Lemma 5.4. Let $\lambda \in \Omega_{2}(G)$. Assume $p>2, L_{G}(\lambda)$ is orthogonal, and moreover the multiplicity of the 0 weight in $L_{G}(\lambda)$ is at most 2. Then $\operatorname{dim} L_{G}(\lambda)$ is even if and only if the pair $(G, \lambda)$ is as in Table 5 .

We give the odd-dimensional orthogonal representations $L_{G}(\lambda)$, with $\lambda \in$ $\Omega_{1}(G)$ in Table 6 .

Lemma 5.5. Let $\lambda \in \Omega_{2}(G)$ such that $L_{G}(\lambda)$ is self-dual and the multiplicity of the 0 weight in $L_{G}(\lambda)$ is at most 2 . Assume in addition that $p=2$. Then $L_{G}(\lambda)$ has a non-degenerate $G$-invariant quadratic form if and only if $(G, \lambda)$ are as in Table [7.

Proof. We first inspect the last column of Table 2, where the dimension of the 0 weight space in $L_{G}(\lambda)$ is given. In addition, the result of Proposition 5.1, and the remarks preceding the proposition, further restrict the list of pairs $(G, \lambda)$ which must be considered. We find that the only even-dimensional $L_{G}(\lambda)$ in addition to those listed in Table 7 are as follows:

a) $G=A_{1}, \lambda=\omega_{1}, \operatorname{dim} L_{G}(\lambda)=2$;

b) $G=C_{n}, \lambda=\omega_{1}, \operatorname{dim} L_{G}(\lambda)=2 n$;

c) $G=G_{2}, \lambda=\omega_{1}, \operatorname{dim} L_{G}(\lambda)=6$. 


\begin{tabular}{|l|c|c|c|}
\hline$G$ & $\lambda$ & conditions & $\operatorname{dim} L_{G}(\lambda)$ \\
\hline$A_{1}$ & $a \omega_{1}$ & $a>0$ even & $a+1$ \\
\hline$A_{n}$ & $(p-1) \omega_{(n+1) / 2}$ & $n>1, n$ odd & not known \\
& $\frac{p-1}{2}\left(\omega_{n / 2}+\omega_{(n+2) / 2}\right)$ & $n$ even & not known \\
\hline$B_{n}$ & $\omega_{1}$ & $n>2$ & $2 n+1$ \\
\hline$C_{2}$ & $\omega_{2}$ & $n \geq 2, \frac{n(p-1)}{2}$ odd & $\left(p^{n}-1\right) / 2$ \\
\hline$C_{n}$ & $\omega_{n-1}+\frac{p-3}{2} \omega_{n}$ & $n \geq 2, \frac{n(p-1)}{2}$, even & $\left(p^{n}+1\right) / 2$ \\
& $\frac{p-1}{2} \omega_{n}$ & $p=3$ & 25 \\
\hline$F_{4}$ & $\omega_{4}$ & $p=3$ & 7 \\
\hline$G_{2}$ & $\omega_{1}$ & & 7 \\
& $\omega_{2}$ & &
\end{tabular}

Table 6: $\lambda \in \Omega_{1}(G), p \neq 2, L_{G}(\lambda)$ odd-dimensional orthogonal

\begin{tabular}{|l|c|c|c|}
\hline$G$ & $\lambda$ & conditions & $\operatorname{dim} L_{G}(\lambda)$ \\
\hline$A_{n}$ & $\omega_{(n+1) / 2}$ & $n>1$ odd & $\left(\begin{array}{c}n+1 \\
\frac{n+1}{2}\end{array}\right)$ \\
\hline$A_{2}$ & $\omega_{1}+\omega_{2}$ & & 8 \\
\hline$A_{3}$ & $\omega_{1}+\omega_{3}$ & & 14 \\
\hline$C_{n}$ & $\omega_{n}$ & & $2^{n}$ \\
\hline$C_{3}$ & $\omega_{2}$ & & 14 \\
\hline$C_{4}$ & $\omega_{2}$ & & 26 \\
\hline$D_{n}$ & $\omega_{1}$ & $n>3$ & $2 n$ \\
\hline$D_{n}$ & $\omega_{n-1}, \omega_{n}$ & $n>3$ even & $2^{n-1}$ \\
\hline$D_{4}$ & $\omega_{2}$ & & 26 \\
\hline$E_{7}$ & $\omega_{7}$ & & 56 \\
\hline$F_{4}$ & $\omega_{1}, \omega_{4}$ & & 26 \\
\hline$G_{2}$ & $\omega_{2}$ & & 14 \\
\hline
\end{tabular}

Table $7: \lambda \in \Omega_{2}(G), p=2, L_{G}(\lambda)$ orthogonal, with 0 weight of multiplicity at most 2 


\begin{tabular}{|l|c|c|}
\hline$G$ & $\lambda$ & conditions \\
\hline$A_{n}$ & $a \omega_{1}, b \omega_{n}$ & $n>1,1 \leq a, b<p$ \\
& $\omega_{i}$ & $1<i<n, i \neq(n+1) / 2$ if $n$ is odd \\
& & \\
& $c \omega_{i}+(p-1-c) \omega_{i+1}$ & $1 \leq i<n, 0 \leq c \leq p-1$, and \\
& & $c \neq(p-1) / 2$ if $n$ is even and $i=n / 2 ;$ \\
& & $c \neq 0$ if $n$ is odd and $i=(n-1) / 2 ;$ \\
& & $c \neq p-1$ if $n$ is odd and $i=(n+1) / 2$. \\
\hline$D_{n}$ & $\omega_{n-1}, \omega_{n}$ & $n>3$ odd \\
\hline$E_{6}$ & $\omega_{1}, \omega_{6}$ & \\
\hline
\end{tabular}

Table 8: $\lambda \in \Omega_{1}(G), \lambda \neq-w_{0} \lambda$ and $\phi(G)$ contains a regular torus of $\operatorname{SL}\left(L_{G}(\lambda)\right)$

\begin{tabular}{|l|c|c|c|}
\hline$G$ & $\lambda$ & conditions & $\operatorname{dim} L_{G}(\lambda)$ \\
\hline$C_{n}$ & $\omega_{1}$ & $n \geq 1$ & $2 n$ \\
\hline$G_{2}$ & $\omega_{1}$ & & 6 \\
\hline
\end{tabular}

Table 9: $p=2, \lambda \in \Omega_{1}(G), L_{G}(\lambda)$ non-orthogonal symplectic

In cases (a), (b) and (c) above, it is well-known that $G$ preserves no nondegenerate quadratic form on $L_{G}(\lambda)$. Hence we now turn to the list of pairs $(G, \lambda)$ of Table 7 . The pair $\left(D_{n}, \omega_{1}\right)$ is clear as $L_{D_{n}}\left(\omega_{1}\right)$ is the natural representation of the classical group of type $D_{n}$.

For cases where $L_{G}(\lambda)$ occurs as a composition factor of the adjoint representation of $G$, we refer to GW95, §3] to conclude that $G$ preserves a nondegenerate quadratic form on $L_{G}(\lambda)$. (Note that we may also apply the exceptional isogeny to the group $F_{4}$. $)$ The pairs $\left(C_{3}, \omega_{2}\right),\left(C_{4}, \omega_{2}\right),\left(E_{7}, \omega_{7}\right)$ are covered in [Bru00, Table 2]. This leaves us with the pairs $\left(A_{n}, \omega_{(n+1) / 2}\right),\left(C_{n}, \omega_{n}\right)$, $\left(D_{n}, \omega_{j}\right), j=n-1, n$. For the second case, we may consider the group $B_{n}$ acting on $L_{G}\left(\omega_{n}\right)$. Then in all cases the Weyl module with the given highest weight is irreducible and the existence of a $G$-invariant quadratic form follows from [SW91, 2.4].

Additionally, we provide in Table 8 the list of modules $L_{G}(\lambda)$, with $\lambda \in$ $\Omega_{1}(G), L_{G}(\lambda) \nRightarrow L_{G}(\lambda)^{*}$. So in particular, the image of $G$ under the corresponding representation contains a regular torus of $H=\mathrm{SL}\left(L_{G}(\lambda)\right)$ and $G$ does not lie in a proper classical subgroup of $H$. Finally, Table 9 records the non-orthogonal symplectic modules $L_{G}(\lambda)$ for $\lambda \in \Omega_{1}(G)$ when $p=2$.

We can now give an explicit solution to Problem 1 for simple subgroups $G$ of classical groups. In Proposition 5.6, we treat the case of tensor-indecomposable irreducible representations of $G$ all of whose weight spaces are 1-dimensional. As discussed in Section 2, the image of $G$ under a tensor-decomposable repre- 
sentation is not maximal in the classical group. In Proposition 5.7, we handle the orthogonal irreducible representations of $G$ whose zero weight space has dimension 2 while all other weight spaces are 1-dimensional. In the following two Propositions, we determine whether the image of $G$ under the given representation is a maximal subgroup of the minimal classical group containing it.

Proposition 5.6. Let $G$ be a simple algebraic group and let $\lambda \in \Omega_{1}(G)$. Let $\rho$ be an irreducible representation of $G$ with highest weight $\lambda$. Then $\rho(G)$ is a maximal subgroup in the minimal classical group containing $\rho(G)$, except for the following cases:

(1) $G=A_{1}, \lambda=6 \omega_{1}, p \geq 7$, where there is an intermediate subgroup of type $G_{2}$

(2) $G=A_{2}, \lambda=\omega_{1}+\omega_{2}, p=3$, where there is an intermediate subgroup of type $G_{2}$;

(3) $G=B_{n}, \lambda=\omega_{n}, p \neq 2$, where there is an intermediate subgroup of type $D_{n+1}$;

(4) $G=C_{n}, \lambda=\omega_{n}, p=2$, where there is an intermediate subgroup of type $D_{n+1}$.

Proof. This follows from Seitz's classification of maximal closed connected subgroups of the classical type simple algebraic groups, see [Sei87, Thm. 3, Table 1] and our results above.

Proposition 5.7. Let $\lambda$ be a weight of $G$ occurring in Tables 5 or 7 but not in Table 1. Let $\rho$ be an irreducible representation with highest weight $\lambda$. In particular, $\rho(G)$ lies in a classical group of type $D_{m}$, and does not contain a regular torus of $\operatorname{SL}\left(L_{G}(\lambda)\right)$. Then $\rho(G)$ is a maximal subgroup of the orthogonal group containing $\rho(G)$, except for the following cases:

(1) $G=A_{3}, \lambda=\omega_{1}+\omega_{3}, p=2$, where there is an intermediate subgroup of type $C_{3}$;

(2) $G=D_{4}, \lambda=\omega_{2}, p=2$, where there are intermediate subgroups of type $C_{4}$ and $F_{4}$;

(3) $G=G_{2}, \lambda=\omega_{2}, p=2$, where there is an intermediate subgroup of type $C_{3}$.

Proof. The proof is carried out exactly as the proof of Proposition 5.6.

\section{Maximal reductive subgroups of exceptional groups containing regular tori}

In this section, we consider Problem 1 for the case where $H$ is an exceptional type simple algebraic group over $F$. We will determine all maximal closed connected subgroups $M$ of $H$ which contain a regular torus. As discussed in Section 2 , we will assume $M$ to be reductive and $\operatorname{rank}(M)<\operatorname{rank}(H)$. The main tool is the classification of the maximal closed connected subgroups of $H$, as given in [LS04, Cor. 2(ii)]. 


\begin{tabular}{|l|l|l|}
\hline$H$ & $M$ simple & Mnon simple \\
\hline \hline$G_{2}$ & $A_{1}(p \geq 7)$ & \\
\hline$F_{4}$ & $A_{1}(p \geq 13), G_{2}(p=7)$ & $A_{1} G_{2}(p \geq 3)$ \\
\hline$E_{6}$ & $A_{2}(p \geq 5), G_{2}(p \neq 7)$ & $A_{2} G_{2}$ \\
& $C_{4}(p \geq 3), F_{4}$ & \\
\hline$E_{7}$ & $A_{1}(p \geq 19), A_{2}(p \geq 5)$ & $A_{1} A_{1}(p \geq 5), A_{1} G_{2}(p \geq 3)$ \\
& & $A_{1} F_{4}, G_{2} C_{3}$ \\
\hline$E_{8}$ & $A_{1}(p \geq 31)$ & $A_{1} A_{2}(p \geq 5), G_{2} F_{4}$ \\
\hline
\end{tabular}

Table 10: Maximal connected reductive subgroups $M \subset H, H$ exceptional, $\operatorname{rank}(M)<\operatorname{rank}(H)$, with $M$ containing a regular torus of $H$

For a semisimple group $M=M_{1} M_{2} \cdots M_{t}$, with $M_{i}$ simple, and with respect to a fixed maximal torus $T_{M}$ of $M$, we will write $\left\{\omega_{i 1}, \ldots, \omega_{i \ell_{i}}\right\}$, for the set of fundamental dominant weights of $T_{M} \cap M_{i}$ (so $\operatorname{rank}\left(M_{i}\right)=\ell_{i}$ ). In case $M$ is simple, we will simply write $\left\{\omega_{1}, \ldots, \omega_{\ell}\right\}$.

Proposition 6.1. Let $M$ be a maximal closed connected positive-dimensional subgroup of an exceptional algebraic group $H$. Assume $\operatorname{rank}(M)<\operatorname{rank}(H)$. Then $M$ contains a regular torus of $H$ if and only if the pair $(M, H)$ is as given in Table 10. In particular, if $M$ contains a regular torus of $H$, then $M$ is semisimple.

Before proving the result, it is interesting to compare the above table with [LS04, Thm. 1], which describes the maximal closed connected positive-dimensional subgroups of the exceptional simple algebraic groups. There are precisely four pairs $(M, H), M$ a maximal closed connected positive-dimensional subgroup of an exceptional algebraic group $H$ with $\operatorname{rank}(M)<\operatorname{rank}(H)$, and where $M$ does not contain a regular torus of $H$ : one class of $A_{1}$ subgroups in $H=E_{7}, 2$ classes of $A_{1}$ subgroups in $H=E_{8}$ and a maximal $B_{2}$ in $E_{8}$.

Proof. Let $M$ be as in the statement of the result and fix $T_{M}$, a maximal torus of $M$. (Throughout the proof we will refer to $M$ as a maximal subgroup, even though $M$ may only be maximal among connected subgroups.) Then LS04, Cor. 2] implies that $M$ is semisimple. The method of proof is quite simple. By Proposition 1.4. $T_{M}$ is a regular torus in $H$ if and only if $\operatorname{dim}\left(C_{\operatorname{Lie}(H)}\left(T_{M}\right)\right)=$ $\operatorname{rank}(H)$. Hence, we need only determine the dimension of the 0 weight space for $T_{M}$ acting on $\operatorname{Lie}(H)$. This can be deduced from the information in [LS04, Table 10.1].

If $M$ is of type $A_{1}$, the notation $T\left(m_{1} ; m_{2} ; \ldots ; m_{k}\right)$, used in [LS04, Table 10.1], represents an $F M$-module whose composition factors are the same as those of $W_{M}\left(m_{1} \omega_{1}\right) \oplus \cdots \oplus W_{M}\left(m_{k} \omega_{1}\right)$. Since the multiplicity of the 0 weight in each Weyl module for $A_{1}$ is precisely 1 , we see that the only maximal $A_{1}$ subgroups containing a regular torus are those listed above. This covers the case $H=G_{2}$. 
Consider now the two remaining cases in $H=F_{4}$. If $M$ is the maximal $G_{2}$ subgroup in $H$ (occurring only for $p=7$ ), then $\left.\operatorname{Lie}(H)\right|_{M}$ has composition factors $L_{M}\left(\omega_{2}\right)$ and $L_{M}\left(\omega_{1}+\omega_{2}\right)$. Now consulting [Lüb, we see that the 0 weight has multiplicity 2 in each of these irreducible modules and hence multiplicity 4 in $\operatorname{Lie}(H)$. This then implies that $T_{M}$ is a regular torus in $H$. For the semisimple subgroup $M=A_{1} G_{2}$ in $F_{4}$ (which exists when $p \geq 3$ ), we must explain an additional notation used in [LS04]. In [LS04, Table 10.1], the notation $\Delta\left(\mu_{1} ; \mu_{2}\right)$ denotes a certain indecomposable $F M$-module whose composition factors are $L_{M}\left(\mu_{1}\right), L_{M}\left(\mu_{2}\right)$ and two factors $L_{M}(\nu)$, where $\mu_{1}$ and $\mu_{2}$ are dominant weights such that the tilting modules $T\left(\mu_{1}\right)$ and $T\left(\mu_{2}\right)$ each have socle and irreducible quotient of highest weight $\nu$. Now if $p>3$, $\left.\operatorname{Lie}(H)\right|_{M}$ has composition factors $L_{M}\left(4 \omega_{11}+\omega_{21}\right), L_{M}\left(2 \omega_{11}\right)$, and $L_{M}\left(\omega_{22}\right)$; the multiplicity of the 0 weight in these modules is $1,1,2$, respectively, and hence $T_{M}$ is a regular torus of $H$. In case $p=3$, the composition factors are $L_{M}\left(4 \omega_{11}+\omega_{21}\right), L_{M}\left(\omega_{22}\right) L_{M}\left(\omega_{21}\right)$, $L_{M}\left(\omega_{21}\right)$, and $L_{M}\left(2 \omega_{11}\right)$, and again the multiplicity of the 0 weight is 4 . This completes the consideration of the case $H=F_{4}$.

Now we consider the case $H=E_{6}$ and $\operatorname{rank}(M) \geq 2$. There is a maximal $A_{2}$ subgroup $M$ of $H$ (when $p \geq 5$ ) whose action on $\operatorname{Lie}(H)$ is $\left.\operatorname{Lie}(H)\right|_{M}=L_{M}\left(4 \omega_{1}+\right.$ $\left.\omega_{2}\right) \oplus L_{M}\left(\omega_{1}+4 \omega_{2}\right) \oplus L_{M}\left(\omega_{1}+\omega_{2}\right)$. Consulting [Lüb], we see that the multiplicity of the zero weight in this module is 6 and so $M$ contains a regular torus of $H$. The group $H$ also has a maximal $G_{2}$ subgroup $M$ when $p \neq 7$, such that $\left.\operatorname{Lie}(H)\right|_{M}$ has the same set of composition factors as $W_{M}\left(\omega_{1}+\omega_{2}\right) \oplus W_{M}\left(\omega_{2}\right)$. Now we consult [Lüb] and find that the multiplicity of the zero weight in $\left.\operatorname{Lie}(H)\right|_{M}$ is 6 for all characteristics $p \neq 7$; hence $M$ contains a regular torus of $H$.

Turn now to the maximal closed connected subgroups of $H=E_{6}$, of rank at least 4 . The maximal subgroup $M$ of type $C_{4}$ acts on $\operatorname{Lie}(H)$ with composition factors $L_{M}\left(2 \omega_{1}\right)$ and $L_{M}\left(\omega_{4}\right)$, if $p \neq 3$, and with these same composition factors plus an additional 1-dimensional composition factor, if $p=3$. As usual, we find that the dimension of the 0 weight space is 6 and so $C_{4}$ contains a regular torus of $H$. For the maximal $F_{4}$ subgroup $M$ of $H$, which exists in all characteristics, $\left.\operatorname{Lie}(H)\right|_{M}=L_{M}\left(\omega_{4}\right) \oplus L_{M}\left(\omega_{1}\right)$, if $p>2$, and when $p=2$, the Lie algebra is isomorphic to the tilting module of highest weight $\omega_{1}$, which has a composition factor $L_{M}\left(\omega_{1}\right)$ and two factors $L_{M}\left(\omega_{4}\right)$. The usual argument shows that $M$ contains a regular torus of $H$. Finally, we consider the maximal subgroup $M \subset H$ of type $A_{2} G_{2} ;\left.\operatorname{Lie}(H)\right|_{M}$ has the same set of $T_{M}$-weights (and multiplicities) as the $F M$-module $W_{M}\left(\omega_{11}+\omega_{12}+\omega_{21}\right) \oplus W_{M}\left(\omega_{11}+\omega_{12}\right) \oplus W_{M}\left(\omega_{22}\right)$. One checks as usual that the multiplicity of the 0 weight is indeed 6 .

We now turn to the case $H=E_{7}$, and $M$ is a maximal closed connected subgroup of rank 2. There exists a maximal $A_{2}$ subgroup $M$ of $H$ when $p \geq 5$, whose action on $\operatorname{Lie}(H)$ is given by $L_{M}\left(4 \omega_{1}+4 \omega_{2}\right) \oplus L_{M}\left(\omega_{1}+\omega_{2}\right)$, when $p \neq 7$, and $\left.\operatorname{Lie}(H)\right|_{M}=T\left(4 \omega_{1}+4 \omega_{2}\right)$, when $p=7$. Again using [Lüb] one verifies the multiplicity of the 0 weight in $\left.\operatorname{Lie}(H)\right|_{M}$ is 7 and hence $T_{M}$ is a regular torus of $H$. The maximal $A_{1} A_{1}$ subgroup $M$ of $H$ is such that $\left.\operatorname{Lie}(H)\right|_{M}$ has the same set of $T_{M}$-weights (and multiplicities) as the module $W_{M}\left(2 \omega_{11}+8 \omega_{21}\right) \oplus$ $W_{M}\left(4 \omega_{11}+6 \omega_{21}\right) \oplus W_{M}\left(6 \omega_{11}+4 \omega_{21}\right) \oplus W_{M}\left(2 \omega_{11}+4 \omega_{21}\right) \oplus W_{M}\left(4 \omega_{11}+2 \omega_{21}\right) \oplus$ 
$W_{M}\left(2 \omega_{11}\right) \oplus W_{M}\left(2 \omega_{21}\right)$. One verifies that the multiplicity of the zero weight is 7 and hence $M$ contains a regular torus of $H$. This completes the consideration of the rank two reductive maximal connected subgroups.

We now handle the remaining maximal connected subgroups of $H=E_{7}$. The maximal $A_{1} G_{2}$ subgroup $M$ of $H$, which exists for all $p \geq 3$, satisfies: Lie $\left.(H)\right|_{M}$ has the same set of $T_{M}$-weights (and multiplicities) as the $F M$-module $W_{M}\left(4 \omega_{11}+\omega_{21}\right) \oplus W_{M}\left(2 \omega_{11}+2 \omega_{21}\right) \oplus W_{M}\left(2 \omega_{11}\right) \oplus W_{M}\left(\omega_{22}\right)$. As usual, we check that the multiplicity of the zero weight in $\left.\operatorname{Lie}(H)\right|_{M}$ is 7 . The maximal $A_{1} F_{4}$ subgroup $M$ is such that $\left.\operatorname{Lie}(H)\right|_{M}$ has the same set of $T_{M}$-weights (and multiplicities) as the module $W_{M}\left(2 \omega_{11}+\omega_{24}\right) \oplus W_{M}\left(2 \omega_{11}\right) \oplus W_{M}\left(\omega_{21}\right)$. This module has a 7 -dimensional 0 weight space and so $M$ contains a regular torus of $H$. Finally, we consider the maximal $G_{2} C_{3}$ subgroup $M$, which exists in all characteristics. In this case, $\left.\operatorname{Lie}(H)\right|_{M}$ has the same set of $T_{M}$-weights (and multiplicities) as the $F M$-module $W_{M}\left(\omega_{11}+\omega_{22}\right) \oplus W_{M}\left(\omega_{12}\right) \oplus W_{M}\left(2 \omega_{21}\right)$, which has a 7-dimensional 0 weight space and again $M$ contains a regular torus of $H$.

To complete the proof, we now turn to the case $H=E_{8}$ and $M$ a maximal closed connected subgroup of rank at least 2 . There exists a unique (up to conjugacy) rank 2 reductive maximal subgroup of $H$, namely $M=B_{2}$, when $p \geq 5$. Here $\left.\operatorname{Lie}(H)\right|_{M}$ has the same set of $T_{M}$-weights (and multiplicities) as $W_{M}\left(6 \omega_{2}\right) \oplus W_{M}\left(3 \omega_{1}+2 \omega_{2}\right) \oplus W_{M}\left(2 \omega_{2}\right)$; but this latter has a 12-dimensional 0 weight space and so $T_{M}$ is not a regular torus of $H$. We now consider the group $M=A_{1} A_{2}$. Here Lie $\left.(H)\right|_{M}$ has the same set of $T_{M}$-weights (and multiplicities) as the $F M$-module $W_{M}\left(6 \omega_{11}+\omega_{21}+\omega_{22}\right) \oplus W_{M}\left(2 \omega_{11}+2 \omega_{21}+2 \omega_{22}\right) \oplus$ $W_{M}\left(4 \omega_{11}+3 \omega_{21}\right) \oplus W_{M}\left(4 \omega_{11}+3 \omega_{22}\right) \oplus W_{M}\left(2 \omega_{11}\right) \oplus W_{M}\left(\omega_{21}+\omega_{22}\right)$, which has an 8-dimensional 0 weight space and hence $M$ contains a regular torus of $H$. Finally, we consider the maximal $G_{2} F_{4}$ subgroup $M$, which exists in all characteristics. Here $\left.\operatorname{Lie}(H)\right|_{M}$ has the same set of $T_{M}$-weights (and multiplicities) as the $F M$-module $W_{M}\left(\omega_{11}+\omega_{24}\right) \oplus W_{M}\left(\omega_{12}\right) \oplus W_{M}\left(\omega_{21}\right)$, which has an 8-dimensional 0 weight space and so $T_{M}$ is a regular torus of $H$.

\section{Non-semisimple regular elements}

In this section, we prove Theorem 1.9, and therefore reduce Problem 1 to the consideration of semisimple elements, the case which has been discussed in detail in the preceding sections. Recall that throughout the paper we are assuming $p \neq 2$ when $G$ is a simple group of type $B_{n}$.

The proof of the following Lemma was provided by Iulian Simion. Our original proof was based upon the classification result of Theorem 7.2, and required a case-by-case analysis. We are very thankful to I. Simion for suggesting this more elegant proof.

Proposition 7.1. Let $G$ be a closed connected reductive subgroup of a reductive algebraic group $H$. Suppose that $G$ contains a regular unipotent element of $H$. Then every maximal torus in $G$ is a regular torus in $H$. 
Proof. Let $T_{G}$ be a maximal torus of $G$. Then $C_{H}\left(T_{G}\right)$ is a Levi subgroup of $H$. (See for example [MT11, Prop.12.10].) Let $B_{H}=T_{H} R_{u}\left(B_{H}\right)$ be a Borel subgroup of $H$ such that $P=\left\langle B_{H}, C_{H}\left(T_{G}\right)\right\rangle$ is a parabolic subgroup of $H$ and $T_{G} \subset T_{H}$. In this proof, we will write $g \cdot x$ to denote the action of $g$ on $x$ by conjugation, that is $g \cdot x=g x g^{-1}$. By assumption, $G$ contains a regular unipotent element of $H$ and [TZ13, Lemma 2.1] then implies that every regular unipotent element of $G$ is regular in $H$. Semisimple elements are dense in $G$ and hence $\overline{G \cdot T_{G}}$ contains a regular unipotent element of $G$, and hence a regular unipotent element of $H$. Now since $H / B_{H}$ is complete, [Ste74, 2.13, Lemma 2] implies that for any closed $B_{H}$-invariant subset $X \subset H, H \cdot X$ is closed. So in particular, since $B_{H} \cdot T_{G}$ is $B_{H}$-stable, so is the closed set $\overline{B_{H} \cdot T_{G}}$ and so $H \cdot \overline{B_{H} \cdot T_{G}}$ is closed and we have

$$
\overline{G \cdot T_{G}} \subset \overline{H \cdot T_{G}}=\overline{H \cdot\left(B_{H} \cdot T_{G}\right)} \subset H \cdot \overline{B_{H} \cdot T_{G}} .
$$

On the other hand, $\overline{B_{H} \cdot T_{G}}$ lies in the radical of $P$, and so any unipotent class intersecting $H \cdot \overline{B_{H} \cdot T_{G}}$ is represented in $R_{u}(P)$. Since $\overline{G \cdot T_{G}}$ contains regular unipotent elements of $H$, we see that $P=B_{H}$ and so $C_{H}\left(T_{G}\right)=T_{H}$ and $T_{G}$ is a regular torus of $H$.

Proof of Theorem 1.9, Let $g \in G$ be a regular element of $H$. Then $g=s u$ where $s \in G$ is semisimple, $u \in G$ is unipotent and $s u=u s$. Set $Y=C_{H}(s)^{\circ}$ and $X=C_{G}(s)^{\circ}$. We first claim that $u$ is a regular unipotent element of $Y$. (See [SS70, Cor. 4.4].) Indeed, if $u$ is not regular in $Y$ then $\operatorname{dim} C_{H}(g)=\operatorname{dim} C_{Y}(u)>$ $\operatorname{rank}(Y)=\operatorname{rank}(H)$, contradicting the regularity of $g$.

By Proposition 7.1 applied to the connected reductive groups $X \subset Y$, a maximal torus $T_{X}$ of $X$ is regular in $Y$. Taking $T_{X}$ such that $s \in T_{X}$, we have $C_{H}\left(T_{X}\right) \subset C_{H}(s)^{\circ}=Y$ and so $C_{H}\left(T_{X}\right) \subset C_{Y}\left(T_{X}\right)$, which is a torus. Now apply Lemma 1.7 to obtain the reuslt.

We conclude the paper with some remarks about how one can determine the reductive overgroups of a general regular element, neither semisimple no unipotent. Let $s u=g \in G \subset H$ be as in the above proof. Then $u$ is a regular unipotent element in the connected reductive group $Y=C_{H}(s)^{\circ}$, lying in the connected reductive group $X=C_{G}(s)^{\circ}$. We now appeal to the following classification:

Theorem 7.2. [TZ13, Theorem 1.4] Let $G$ be a closed semisimple subgroup of the simple algebraic group $H$, containing a regular unipotent element of $H$. Then $G$ is simple and either the pair $(H, G)$ is as given in Table 11, or $G$ is of type $A_{1}$ and $p=0$ or $p \geq h$, where $h$ is the Coxeter number for $H$. Moreover, for each pair of root systems $(\Phi(H), \Phi(G))$ as in the table, respectively, for $\left(\Phi(H), A_{1}, p\right)$, with $p=0$ or $p \geq h$, there exists a closed simple subgroup $X \subset H$ of type $\Phi(G)$, respectively $A_{1}$, containing a regular unipotent element of $H$.

The above classification applies to simple groups $H$, but it is straightforward to deduce from this the set of possible pairs of reductive groups $\left(C_{G}(s)^{\circ}, C_{H}(s)^{\circ}\right)$. 


\begin{tabular}{|l|l|}
\hline$H$ & $G$ \\
\hline$A_{6}$ & $G_{2}, p \neq 2$ \\
$A_{5}$ & $G_{2}, p=2$ \\
\hline$C_{3}$ & $G_{2}, p=2$ \\
\hline$B_{3}$ & $G_{2}, p \neq 2$ \\
\hline$D_{4}$ & $G_{2}, p \neq 2$ \\
& $B_{3}$ \\
\hline$E_{6}$ & $F_{4}$ \\
\hline$A_{n-1}, n>1$ & $C_{n / 2}, n$ even \\
& $B_{(n-1) / 2}, n$ odd, $p \neq 2$ \\
\hline$D_{n}, n>4$ & $B_{n-1}$ \\
\hline
\end{tabular}

Table 11: Semisimple subgroups $G \subset H$ containing a regular unipotent element

Coupling this with information about centralizers of semisimple elements in $H$ and in $G$, we can obtain a classification of the reductive subgroups of $H$ containing $g$.

Remark 7.3. We point out here an inaccuracy in [SS97] and [TZ13]. In the statement of [SS97, Thm. A], the condition given for the existence of an $A_{1}$ subgroup containing a regular unipotent element is $p>h$. But in fact, as shown in [Tes95], such a subgroup exists for all $p \geq h$. This has been correctly stated in Theorem 7.2 ,

\section{References}

[BBL97] Georgia Benkart, Daniel Britten, and Frank Lemire. Modules with bounded weight multiplicities for simple Lie algebras. Math. Z., 225(2):333-353, 1997.

[BGT] Tim Burness, Soumaïa Ghandour, and D.M. Testerman. Irreducible geometric subgroups of classical algebraic groups. Memoirs of the Amer. Math. Soc., to appear.

[BOS08] A. A. Baranov, A. A. Osinovskaya, and I. D. Suprunenko. Modular representations of classical groups with small weight multiplicities. Sovrem. Mat. Prilozh., (60, Algebra):162-174, 2008.

[BOS14] A. A. Baranov, A. A. Osinovskaya, and I. D. Suprunenko. Modular representations of the special linear groups with small weight multiplicities. J. Algebra, 397:225-251, 2014.

[Bou68] N. Bourbaki. Éléments de mathématique. Fasc. XXXIV. Groupes et algèbres de Lie. Chapitre IV: Groupes de Coxeter et systèmes de Tits. Chapitre V: Groupes engendrés par des réflexions. Chapitre VI: 
systèmes de racines. Actualités Scientifiques et Industrielles, No. 1337. Hermann, Paris, 1968.

[Bru00] Jonathan Brundan. Double coset density in classical algebraic groups. Trans. Amer. Math. Soc., 352(3):1405-1436 (electronic), 2000.

[BT71] A. Borel and J. Tits. Éléments unipotents et sous-groupes paraboliques de groupes réductifs. I. Invent. Math., 12:95-104, 1971.

[Coo81a] Bruce N. Cooperstein. Subgroups of exceptional groups of Lie type generated by long root elements. I. Odd characteristic. J. Algebra, 70(1):270-282, 1981.

[Coo81b] Bruce N. Cooperstein. Subgroups of exceptional groups of Lie type generated by long root elements. II. Characteristic two. J. Algebra, 70(1):283-298, 1981.

[GW95] Roderick Gow and Wolfgang Willems. Methods to decide if simple self-dual modules over fields of characteristic 2 are of quadratic type. J. Algebra, 175(3):1067-1081, 1995.

[Her74] Christoph Hering. Transitive linear groups and linear groups which contain irreducible subgroups of prime order. Geometriae Dedicata, 2:425-460, 1974.

[Hum75] James E. Humphreys. Linear algebraic groups. Springer-Verlag, New York, 1975. Graduate Texts in Mathematics, No. 21.

[Kan79] William M. Kantor. Subgroups of classical groups generated by long root elements. Trans. Amer. Math. Soc., 248(2):347-379, 1979.

[LS94] Martin W. Liebeck and Gary M. Seitz. Subgroups generated by root elements in groups of Lie type. Ann. of Math. (2), 139(2):293-361, 1994.

[LS04] Martin W. Liebeck and Gary M. Seitz. The maximal subgroups of positive dimension in exceptional algebraic groups. Mem. Amer. Math. Soc., 169(802):vi+227, 2004.

[Lüb] Frank Lübeck. Tables of weight multiplicities. On line tables. http://www.math.rwth-aachen.de/ Frank.Lübeck/chev/WMSmall/index.html.

[Lüb01] Frank Lübeck. Small degree representations of finite Chevalley groups in defining characteristic. LMS J. Comput. Math., 4:135-169 (electronic), 2001.

[MT11] Gunter Malle and Donna Testerman. Linear algebraic groups and finite groups of Lie type, volume 133 of Cambridge Studies in Advanced Mathematics. Cambridge University Press, Cambridge, 2011. 
[Pol76] Harriet Pollatsek. Irreducible groups generated by transvections over finite fields of characteristic two. J. Algebra, 39(1):328-333, 1976.

[Pre87] A. A. Premet. Weights of infinitesimally irreducible representations of Chevalley groups over a field of prime characteristic. Mat. Sb. (N.S.), 133(175)(2):167-183, 271, 1987.

[PS83] A. A. Premet and I. D. Suprunenko. The Weyl modules and the irreducible representations of the symplectic group with the fundamental highest weights. Comm. Algebra, 11(12):1309-1342, 1983.

[Sei83] Gary M. Seitz. The root subgroups for maximal tori in finite groups of Lie type. Pacific J. Math., 106(1):153-244, 1983.

[Sei87] Gary M. Seitz. The maximal subgroups of classical algebraic groups. Mem. Amer. Math. Soc., 67(365):iv+286, 1987.

[SS70] T. A. Springer and R. Steinberg. Conjugacy classes. In Seminar on Algebraic Groups and Related Finite Groups (The Institute for Advanced Study, Princeton, N.J., 1968/69), Lecture Notes in Mathematics, Vol. 131, pages 167-266. Springer, Berlin, 1970.

[SS97] Jan Saxl and Gary M. Seitz. Subgroups of algebraic groups containing regular unipotent elements. J. London Math. Soc. (2), 55(2):370-386, 1997.

[Ste68] Robert Steinberg. Lectures on Chevalley groups. Yale University, New Haven, Conn., 1968. Notes prepared by John Faulkner and Robert Wilson.

[Ste74] Robert Steinberg. Conjugacy classes in algebraic groups. Lecture Notes in Mathematics, Vol. 366. Springer-Verlag, Berlin, 1974. Notes by Vinay V. Deodhar.

[Sup95] Irina D. Suprunenko. Irreducible representations of simple algebraic groups containing matrices with big Jordan blocks. Proc. London Math. Soc. (3), 71(2):281-332, 1995.

[SW91] Peter Sin and Wolfgang Willems. $G$-invariant quadratic forms. $J$. Reine Angew. Math., 420:45-59, 1991.

[SZ92] Irina D. Suprunenko and A.E. Zalesski. Irreducible representations of finite chevalley groups containing a matrix with a simple spectrum. In Groups, combinatorics \& geometry (Durham, 1990), volume 165 of London Math. Soc. Lecture Note Ser., pages 327-331. Cambridge Univ. Press, Cambridge, 1992.

[SZ98] Irina D. Suprunenko and Alexander E. Zalesskii. Irreducible representations of finite classical groups containing matrices with simple spectra. Comm. Algebra, 26(3):863-888, 1998. 
[SZ00] I. D. Suprunenko and A. E. Zalesskii. Irreducible representations of finite exceptional groups of Lie type containing matrices with simple spectra. Comm. Algebra, 28(4):1789-1833, 2000.

[Tes88] Donna M. Testerman. Irreducible subgroups of exceptional algebraic groups. Mem. Amer. Math. Soc., 75(390):iv+190, 1988.

[Tes95] Donna M. Testerman. $A_{1}$-type overgroups of elements of order $p$ in semisimple algebraic groups and the associated finite groups. J. Algebra, 177(1):34-76, 1995.

[Tho71] J. G. Thompson. Quadratic pairs. In Actes du Congrès International des Mathématiciens (Nice, 1970), Tome 1, pages 375-376. GauthierVillars, Paris, 1971.

[TZ13] Donna Testerman and Alexandre Zalesski. Irreducibility in algebraic groups and regular unipotent elements. Proc. Amer. Math. Soc., 141(1):13-28, 2013.

[Vav90] N. A. Vavilov. Subgroups of Chevalley groups that contain a maximal torus. Trudy Leningrad. Mat. Obshch., 1:64-109, 245-246, 1990.

[Vav95] Nikolai Vavilov. Intermediate subgroups in Chevalley groups. In Groups of Lie type and their geometries (Como, 1993), volume 207 of London Math. Soc. Lecture Note Ser., pages 233-280. Cambridge Univ. Press, Cambridge, 1995.

[Wag78] A. Wagner. Collineation groups generated by homologies of order greater than 2. Geom. Dedicata, 7(4):387-398, 1978.

[Wag80] Ascher Wagner. Determination of the finite primitive reflection groups over an arbitrary field of characteristic not 2. I. Geom. Dedicata, 9(2):239-253, 1980.

[Wag81] Ascher Wagner. Determination of the finite primitive reflection groups over an arbitrary field of characteristic not two. II, III. Geom. Dedicata, 10(1-4):191-203, 475-523, 1981.

[Wal90] John H. Walter. Rigid cyclic subgroups in Chevalley groups. I. J. Algebra, 131(2):688-702, 1990.

[ZS76] A. E. Zalesskiı̌ and V. N. Serežkin. Linear groups generated by transvections. Izv. Akad. Nauk SSSR Ser. Mat., 40(1):26-49, 221, 1976.

[ZS80] A. E. Zalesskiı and V. N. Serežkin. Finite linear groups generated by reflections. Izv. Akad. Nauk SSSR Ser. Mat., 44(6):1279-1307, 38, 1980. 
[ZS87] A. E. Zalesskil and I. D. Suprunenko. Representations of dimension $\left(p^{n} \pm 1\right) / 2$ of the symplectic group of degree $2 n$ over a field of characteristic p. Vestsī Akad. Navuk BSSR Ser. Fīz.-Mat. Navuk, (6):9-15, $123,1987$.

[ZS90] A. E. Zalesskiı̌ and I. D. Suprunenko. Truncated symmetric powers of natural realizations of the groups $\mathrm{SL}_{m}(P)$ and $\operatorname{Sp}_{m}(P)$ and their constraints on subgroups. Sibirsk. Mat. Zh., 31(4):33-46, 220, 1990. 